\title{
Novel Receiver Architecture for LTE-A Downlink Physical Control Format Indicator Channel with Diversity
}

\author{
S. Syed Ameer Abbas, ${ }^{1}$ S. J. Thiruvengadam, ${ }^{2}$ and S. Susithra ${ }^{1}$ \\ ${ }^{1}$ Department of Electronics and Communication Engineering, Mepco Schlenk Engineering College, Sivakasi 626 005, India \\ ${ }^{2}$ Department of Electronics and Communication Engineering, Thiagarajar College of Engineering, Madurai 625 015, India \\ Correspondence should be addressed to S. Syed Ameer Abbas; abbas_mepco@yahoo.com
}

Received 14 November 2013; Revised 27 April 2014; Accepted 5 May 2014; Published 5 June 2014

Academic Editor: Chien-In Henry Chen

Copyright (c) 2014 S. Syed Ameer Abbas et al. This is an open access article distributed under the Creative Commons Attribution License, which permits unrestricted use, distribution, and reproduction in any medium, provided the original work is properly cited.

\begin{abstract}
Physical control format indicator channel (PCFICH) carries the control information about the number of orthogonal frequency division multiplexing (OFDM) symbols used for transmission of control information in long term evolution-advanced (LTEA) downlink system. In this paper, two novel low complexity receiver architectures are proposed to implement the maximum likelihood- (ML-) based algorithm which decodes the CFI value in field programmable gate array (FPGA) at user equipment (UE). The performance of the proposed architectures is analyzed in terms of the timing cycles, operational resource requirement, and resource complexity. In LTE-A, base station and UE have multiple antenna ports to provide transmit and receive diversities. The proposed architectures are implemented in Virtex-6 xc6vlx240tffl156-1 FPGA device for various antenna configurations at base station and UE. When multiple antenna ports are used at base station, transmit diversity is obtained by applying the concept of space frequency block code (SFBC). It is shown that the proposed architectures use minimum number of operational units in FPGA compared to the traditional direct method of implementation.
\end{abstract}

\section{Introduction}

The goal of third generation partnership project (3GPP) long term evolution-advanced (LTE-A) wireless standard is to increase the capacity and speed of wireless data communication. The LTE-A physical layer is a highly efficient means of conveying both data and control information between an enhanced base station, popularly known as eNodeB, and mobile user equipment (UE). It supports both frequency division duplex (FDD) and time division duplex (TDD) configurations in uplink and downlink operations. Further, it provides a wide range of system bandwidths in order to operate in a large number of different spectrum allocations [1].

LTE-A standard has six physical channels for downlink. They are physical broadcast channel $(\mathrm{PBCH})$, physical downlink shared channel (PDSCH), physical multicast channel $(\mathrm{PMCH})$, physical downlink control channel (PDCCH), physical hybrid automatic repeat request (ARQ) indicator channel (PHICH), and physical control format indicator channel (PCFICH). PBCH carries the basic system information for the other channels to be configured and operated in the LTE-A grid. The PDSCH is the main data-bearing channel. PMCH is defined for future use. In LTE-A, the control signals are transmitted at the start of each subframe in the LTE-A grid. PDCCH is used to carry the scheduling information of different types such as downlink resource scheduling and uplink power control instructions. $\mathrm{PHICH}$ is used to send the acknowledgement/negative acknowledgement bit to UEs to indicate whether the uplink user data is correctly received or not. PCFICH carries the control information about the number of orthogonal frequency division multiplexing (OFDM) symbols used for transmission of downlink control information. The high data rate in LTE-A requires high processing demands on all layers of the system which includes high digital signal processing (DSP) hardware processing in the physical layer. Further, the hardware implementation of receiver structures of various physical channels in LTE-A becomes a challenging task as the computational complexity increases. 
In [2], receivers were designed for a $2 \times 2$ antenna system and for quadrature phase shift keying (QPSK) modulation and quadrature amplitude modulation (16-QAM and 64QAM). Though successive interference cancellation (SIC) receiver meets the timing requirements in the LTE system, it is complex and the K-best list sphere detector (K-LSD) receiver has high latency. In [3], field programmable gate array (FPGA) and application specific integrated circuit (ASIC) implementations of receivers based on the linear minimum mean-square error (LMMSE), the K-LSD, iterative successive interference cancellation (SIC) detector, and the iterative K-LSD algorithms are carried out for spatial multiplexing based LTE-A system. The SIC algorithm is found to perform worse than the K-LSD when the MIMO channels are highly correlated, while the performance difference diminishes when the correlation decreases. The ASIC receivers are designed to meet the decoding throughput requirements in LTE and the K-LSD is found to be the most complex receiver although it gives the best reliable data transmission throughput. It is shown that the receiver architecture which could be reconfigured to use a simple or a more complex detector as the channel conditions change would achieve the best performance while consuming the least amount of power in the receiver. FPGA implementation of MIMO detector based on two typical sphere decoding algorithms, namely, the Viterbo-Boutros (VB) algorithm and the Schnorr-Euchner (SE) algorithm, is carried out in [4]. In this implementation method, three levels of parallelism are explored to improve the decoding rate: the concurrent execution of the channel matrix preprocessing on an embedded processor and the decoding functions on customized hardware modules, the parallel decoding of real/imaginary parts for complex constellation, and the concurrent execution of multiple steps during the closest lattice point search. The implementation of low-complexity codebook searching engine is proposed to support both LTE and LTE-A operations [5]. In [6], VLSI implementation of a low-complexity multiple input multiple output (MIMO) symbol detector based on a novel MIMO detection algorithm called modified fixed-complexity soft-output (MFCSO) detection is presented. It includes a microcode-controlled channel preprocessing unit, separate channel memory, and a pipelined detection unit. MATLABbased downlink physical-layer simulator for LTE only for research applications is presented [7]. In [8], maximum likelihood- (ML-) based receiver structures are developed for decoding the downlink control channels PCFICH and $\mathrm{PHICH}$ in LTE wireless standard and the performance of the receivers has been analyzed for various configurations. The analytical results were validated against computer simulations but hardware implementation of the structures was not coded or synthesized. In [9], direct implementation of receive algorithms was carried out in FPGA for downlink control channels in LTE. However, most of these works either propose architectures for FPGA implementation or analyze the performance of various receiver structures in a generalized manner. The objective of this paper is to propose novel architectures for FPGA implementation of transmit and receive processing of downlink PCFICH channel in LTE-A standard in particular.
TABLE 1: CFI 32-bit block code.

\begin{tabular}{lc}
\hline CFI & $\left\langle b_{31}, \ldots, b_{0}\right\rangle$ \\
\hline 1 & 01101101101101101101101101101101 \\
2 & 10110110110110110110110110110110 \\
3 & 11011011011011011011011011011011 \\
4 & 00000000000000000000000000000000 \\
\hline
\end{tabular}

1.1. Transmit and Receive Processing of PCFICH. In PCFICH, the control format indicator (CFI) contains a 32-bit code word that represents the value of CFI as $1,2,3$, or 4 . The CFI informs the UE about the number of OFDM symbols used for the transmission of PDCCH information in a subframe. The 32-bit code word corresponding to the value of CFI is scrambled and QPSK modulated. The resultant 16 QPSK complex symbols are mapped to the resource elements of the first OFDM symbol of every subframe after layer mapping and precoding to obtain transmit diversity when two or more antenna ports are used at eNodeB [10]. The 32-bit code words for the four possible values of CFI are given in Table 1. A general block diagram of the transmitter and receiver processing of PCFICH is shown in Figure 1.

The OFDM signal is transmitted through a frequency selective fading channel. It is assumed that the number of receive antenna ports at $\mathrm{UE}$ is $K$. At each receive antenna port of the UE resource-element demapping follows the cyclic prefix removal and fast fourier transformation (FFT). The $16 \times 1$ receive signal vector at each antenna port is equalized in frequency domain at each subcarrier using the corresponding $16 \times 1$ channel frequency response vector. The outputs of frequency domain equalizer from each antenna port are summed up. The resultant $16 \times 1$ complex vector is applied to the maximum likelihood (ML) detector for detecting the CFI value. The objective of this paper is to synthesize and implement the receiver architecture for PCFICH.

The paper is structured as follows. Section 2 explains the system model and basic implementation architectures for single input single output (SISO) and single input multiple output (SIMO) configurations. The system model and basic implementation architecture for multiple input single output (MISO) and multiple input multiple output (MIMO) configurations are described in Sections 3 and 4, respectively. The proposed implementation architectures using folding and superscalar methods are given in Section 5 for SISO, SIMO, MISO, and MIMO configurations. Section 6 analyzes the performance of the proposed architectures and Section 7 concludes the paper with remarks on future work.

\section{System Model and Implementation Architecture for SISO and SIMO Configurations}

The received signal model for SISO configuration of PCFICH is given by

$$
\mathbf{y}=\mathbf{h} \circ \mathbf{d}^{(m)}+\mathbf{w},
$$




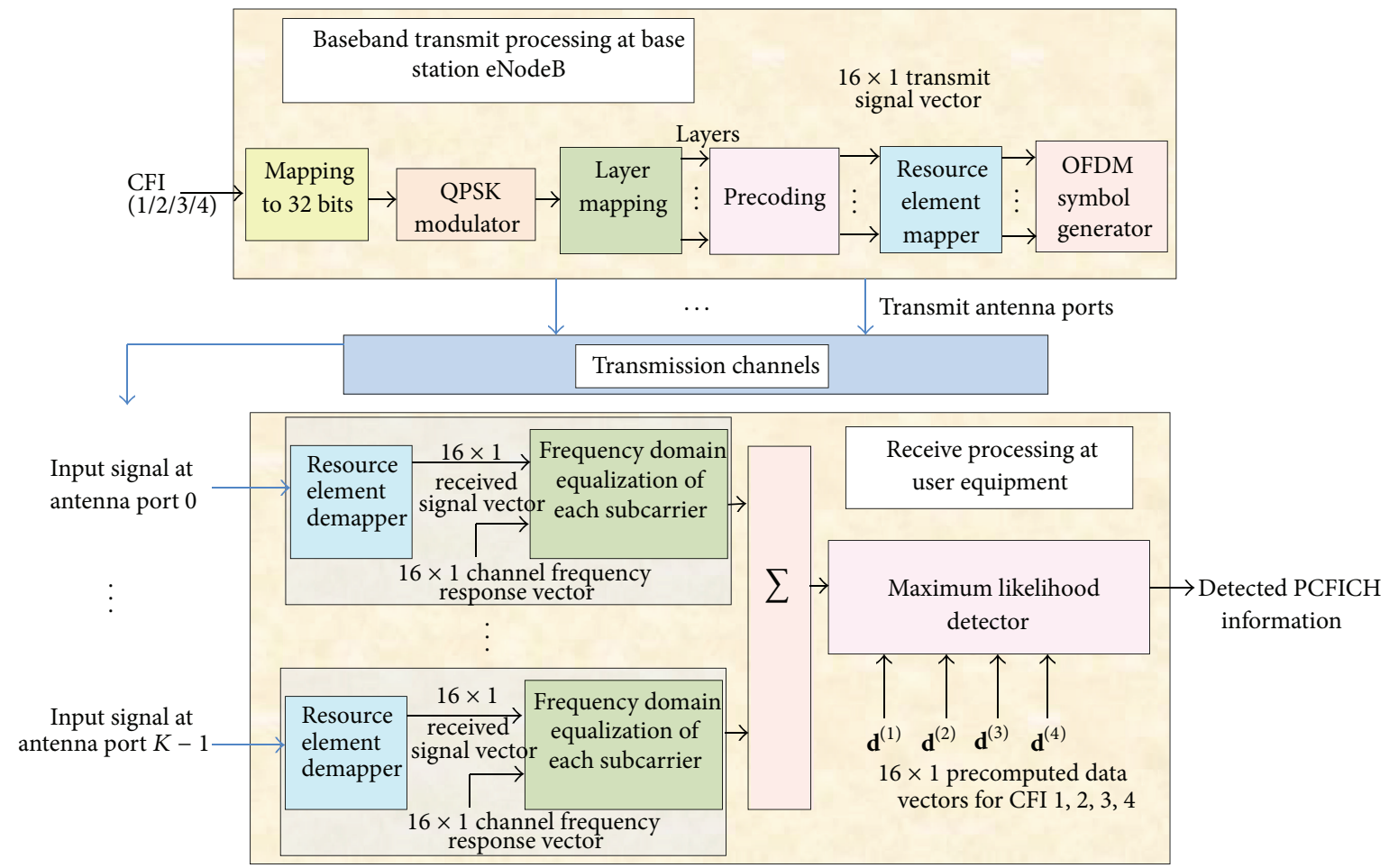

FIGURE 1: Block diagram of transmitter and receiver processing.

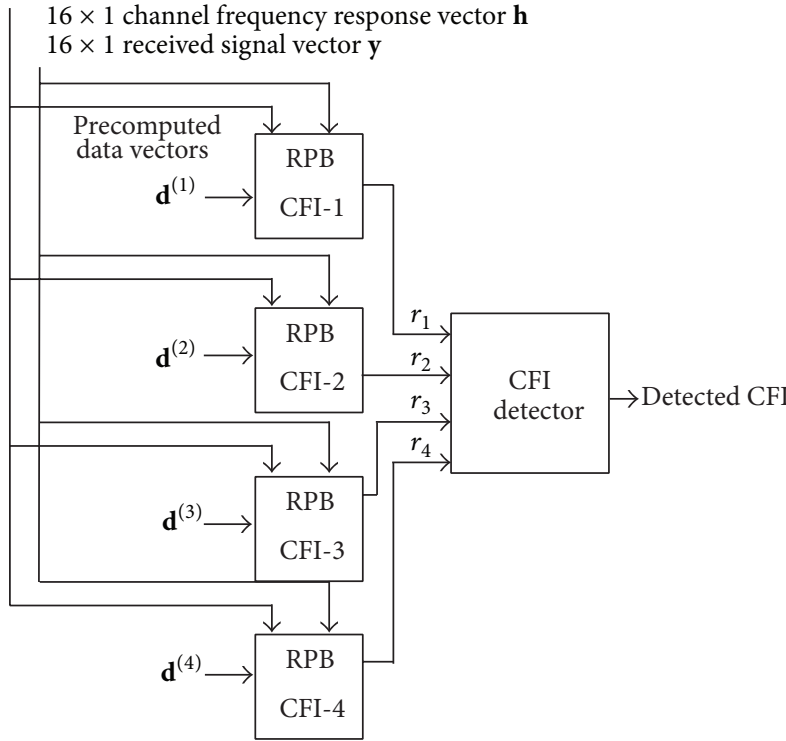

FIGURE 2: Basic architecture for SISO configuration.

where $\mathbf{y}=\left[y_{0}, y_{1}, \ldots, y_{15}\right]^{T}$ is a $16 \times 1$ received signal vector, $\mathbf{h}=\left[h_{0}, h_{1}, \ldots, h_{15}\right]^{T}$ is a $16 \times 1$ channel frequency response vector, $\mathbf{d}^{(m)}=\left[d_{0}^{(m)}, d_{1}^{(m)}, \ldots, d_{15}^{(m)}\right]^{T}$ is a $16 \times 1$ complex QPSK symbol vector corresponding to CFI value from the set $\{1,2,3,4\}$, “” represents the element by element multiplication, and $\mathbf{w}$ is a $16 \times 1$ additive white noise vector and its elements are zero mean Gaussian random numbers with unit variance. The objective is to detect the value of CFI from the received signal vector $\mathbf{y}$ assuming the channel frequency response vector $\mathbf{h}$ to be known. Using maximum likelihood (ML) principle, $\mathrm{CFI}$ is detected as

$$
\widehat{\mathrm{CFI}}=\underset{m}{\operatorname{argmin}}\left\|\mathbf{y}-\mathbf{h} \circ \mathbf{d}^{(m)}\right\|^{2} .
$$

Figure 2 shows the basic architecture for estimating CFI using (2), in SISO configuration. The received signal vector $\mathbf{y}$ and the channel frequency response vector $\mathbf{h}$ are provided as input to the four receiver processing blocks (RPB) along with precomputed data vectors $\mathbf{d}^{(1)}, \mathbf{d}^{(2)}, \mathbf{d}^{(3)}$, and $\mathbf{d}^{(4)}$. The internal diagram for RPB CFI-1 is shown in Figure 3. It computes the expression $\left\|\mathbf{y}-\mathbf{h} \circ \mathbf{d}^{(1)}\right\|^{2}$ assuming the CFI $=1$. In RPB$\mathrm{m}$, the precomputed data vector $\mathbf{d}^{(m)}$ is multiplied element by element with the channel frequency response vector. The resultant $(16 \times 1)$ vector is subtracted from the $(16 \times 1)$ received signal vector $\mathbf{y}$. The sum of squared magnitude of each element in the resultant vector is the output of RPB.

The inputs to the CFI detector are the 16-bit outputs of RPBs $r_{1}, r_{2}, r_{3}$, and $r_{4}$. The CFI detector determines which $\mathrm{RPB}$ output has minimum value. The internal diagram for CFI detector circuit which has 4 comparator modules (CM) is shown in Figure 4. In CM-1, input $r_{2}$ and one's complement 
$16 \times 1$ channel frequency response vector

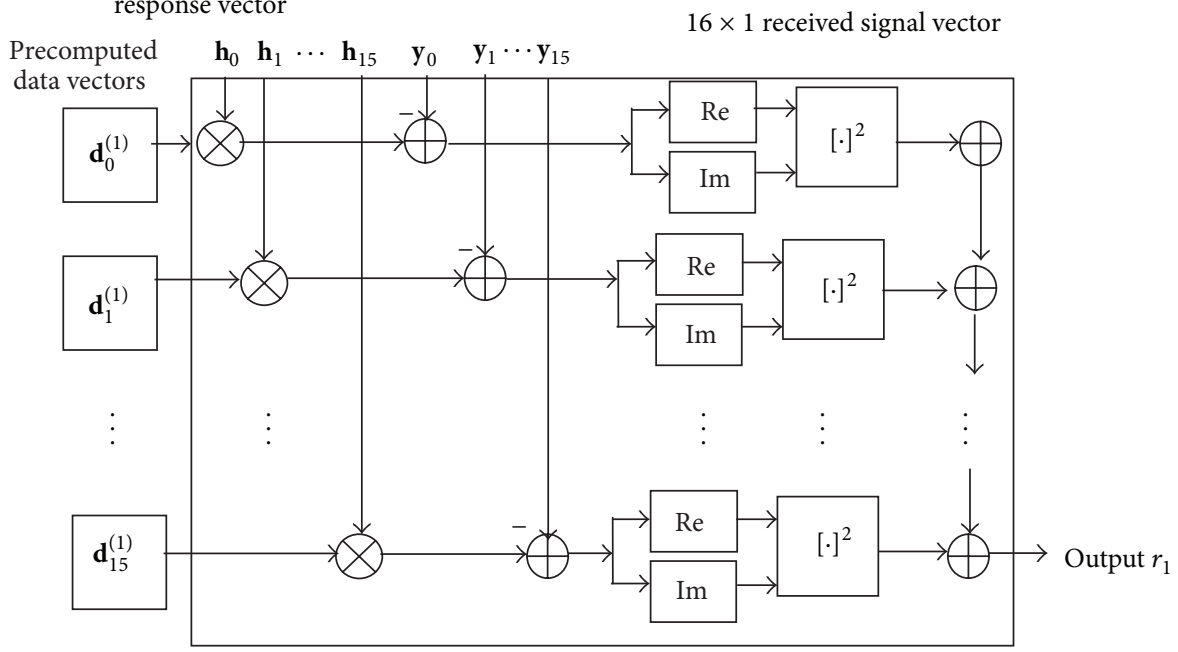

FIGURE 3: Internal architecture of receiver processing block (RPB CFI-1).

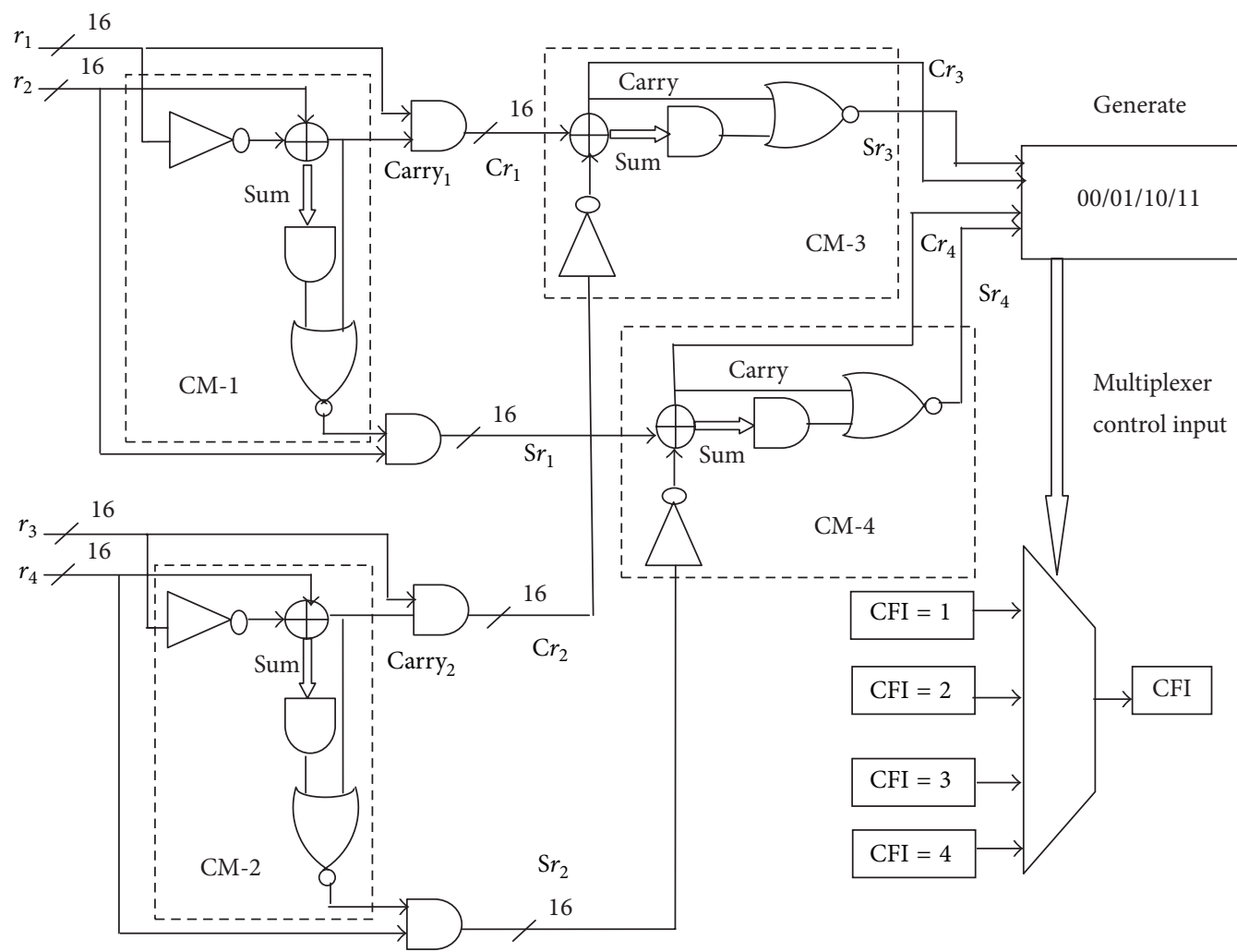

FIGURE 4: Internal architecture of CFI detector.

of input $r_{1}$ are added. If carry is generated, then $r_{1}$ is less than $r_{2}$. The outputs $\mathrm{Cr}_{1}$ and $\mathrm{S} r_{1}$ of the CM-1 are defined as
In CM-2, input $r_{4}$ and one's complement of input $r_{3}$ are added. If carry is generated, then $r_{3}$ is less than $r_{4}$. The outputs $\mathrm{Cr}_{2}$ and $\mathrm{Sr}_{2}$ of CM-2 are defined as

$$
\begin{aligned}
& C r_{1}=\left\{\begin{array}{l}
r_{1} \quad \text { if } \text { carry }_{1}=" 1 "\left(r_{1}<r_{2}\right) \\
0 \quad \text { if carry }=\text { " } 0 "\left(r_{1}>r_{2}\right),
\end{array}\right. \\
& S r_{1}= \begin{cases}r_{2} & \text { if } \text { carry }_{1}=" 0 "\left(r_{1}>r_{2}\right) \\
0 & \text { if carry } \\
1 & =" 1 "\left(r_{1}<r_{2}\right) .\end{cases}
\end{aligned}
$$

$$
\begin{aligned}
& \mathrm{Cr}_{2}= \begin{cases}r_{3} & \text { if } \text { carry }_{2}=" 1 "\left(r_{3}<r_{4}\right) \\
0 & \text { if carry } \\
2 & =" 0 "\left(r_{3}>r_{4}\right),\end{cases} \\
& S r_{2}= \begin{cases}r_{4} & \text { if carry } \\
2 & =" 0 "\left(r_{3}>r_{4}\right) \\
0 & \text { if carry } \\
2 & =" 1 "\left(r_{3}<r_{4}\right) .\end{cases}
\end{aligned}
$$




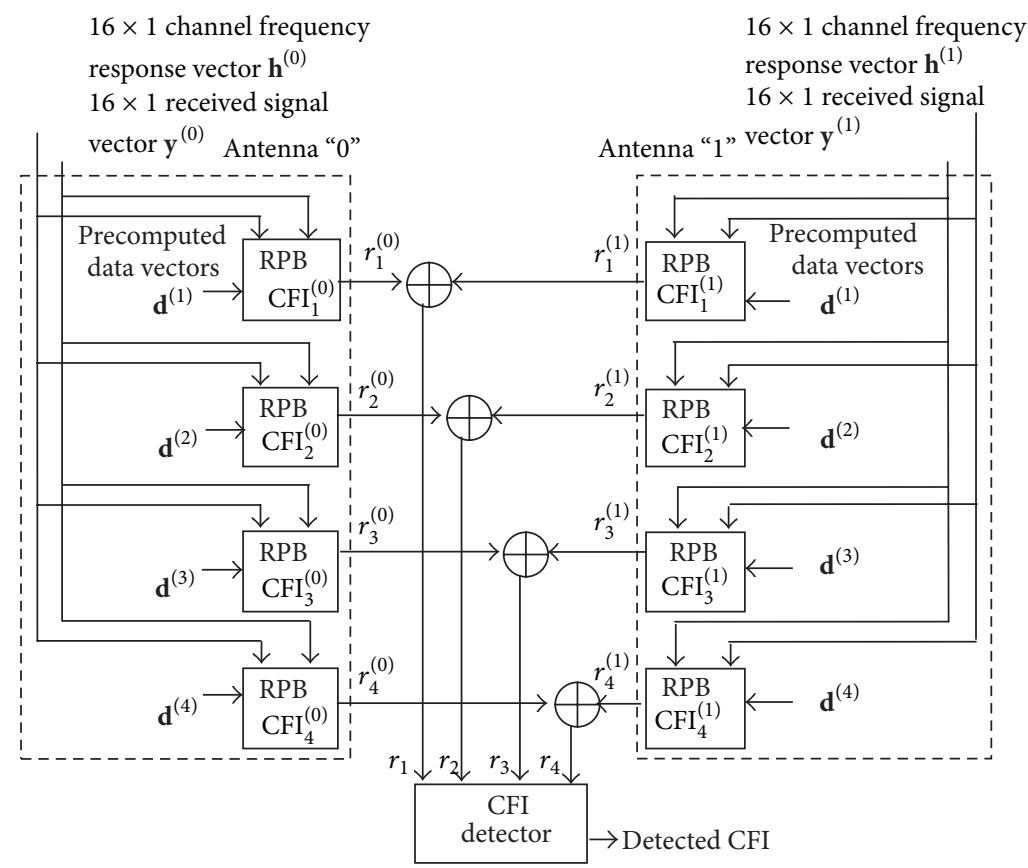

FIgURE 5: Basic architecture for SIMO configuration.

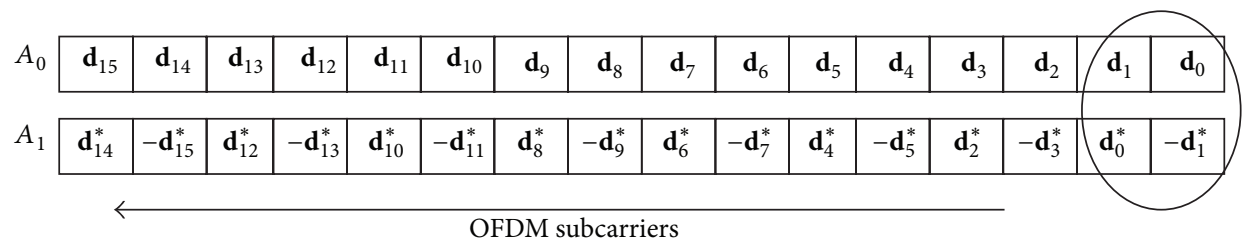

FIGURE 6: Subcarrier mapping for SFBC for 2-antenna system.

The multiplexer control input is activated based on the outputs from CM-3 and CM-4. One of the four outputs $\mathrm{Cr}_{3}$, $\mathrm{Sr}_{3}, \mathrm{Cr}_{4}$, and $\mathrm{Sr}_{4}$ would be "1" based on the minimum value of four inputs $r_{1}, r_{2}, r_{3}$, and $r_{4}$, respectively. Based on this 00 , 01,10 , or 11 in the multiplexer control unit would be activated to obtain the detected CFI value.

In SIMO, the $16 \times 1$ receive signal vector at the $k$ th receive antenna is modeled as

$$
\mathbf{y}^{(k)}=\mathbf{h}^{(k)} \circ \mathbf{d}^{(m)}+\mathbf{w}_{k}, \quad k=0,1,2, \ldots, K-1,
$$

where " $K$ " represents the number of receive antennas at UE, $\mathbf{h}^{(k)}$ is $16 \times 1$ channel frequency response vector between the transmit antenna and $k$ th receive antenna, and $\mathbf{w}_{k}$ is $16 \times 1$ noise vector at $k$ th receive antenna. Now, the objective is to detect the value of CFI from the received signal vectors at each receive antenna, assuming the channel frequency response vectors at each receive antenna are known. The maximal ratio combining is carried out at the receiver. Using maximum likelihood (ML) principle, CFI is estimated as [9]

$$
\widehat{\mathrm{CFI}}=\min _{m=1,2,3,4} \sum_{k=1}^{K}\left\|\mathbf{y}^{(k)}-\left(\mathbf{h}^{(k)} \circ \mathbf{d}^{(m)}\right)\right\|^{2}
$$

The basic architecture for estimating CFI using (6) in $1 \times 2$ SIMO configuration shown in Figure 5 is similar to the basic architecture of SISO configuration. The received signal vector $\mathbf{y}^{(k)}$ and the channel frequency response vector $\mathbf{h}^{(k)}$ are provided as input to the four receiver processing blocks $\left(\mathrm{RPB}-\mathrm{CFI}_{m}^{(k)}\right)$ at $k$ th receive antenna, along with precomputed data vectors $\mathbf{d}^{(1)}, \mathbf{d}^{(2)}, \mathbf{d}^{(3)}$, and $\mathbf{d}^{(4)}$. The outputs from the $m$ th $\mathrm{RPB}$ at 0 th receive antenna $r_{m}^{(0)}$ and 1 st receive antenna $r_{m}^{(1)}$ are added to get the $m$ th input $r_{m}$ of the CFI detector circuit.

\section{System Model and Implementation Architecture for MISO Configuration}

In MISO and MIMO configurations, space frequency block code (SFBC) based layer mapping and precoding are carried out to obtain transmit diversity when two or more antenna ports are used at eNodeB as per the 3GPP LTE wireless standard $[1,11]$. It is assumed that 2 antenna ports are used at $\mathrm{eNodeB}$. The $16 \times 1$ complex symbol vector output of the modulation mapper is applied to the layer mapper. The $8 \times 1$ symbol vectors at layer 0 and layer 1 are given by $\left[d_{0}, d_{2}, d_{4}, d_{6}, d_{8}, d_{10}, d_{12}\right.$, and $\left.d_{14}\right]$ and $\left[d_{1}, d_{3}, d_{5}, d_{7}\right.$, $d_{9}, d_{11}, d_{13}$, and $d_{15}$. The precoding is carried out using 


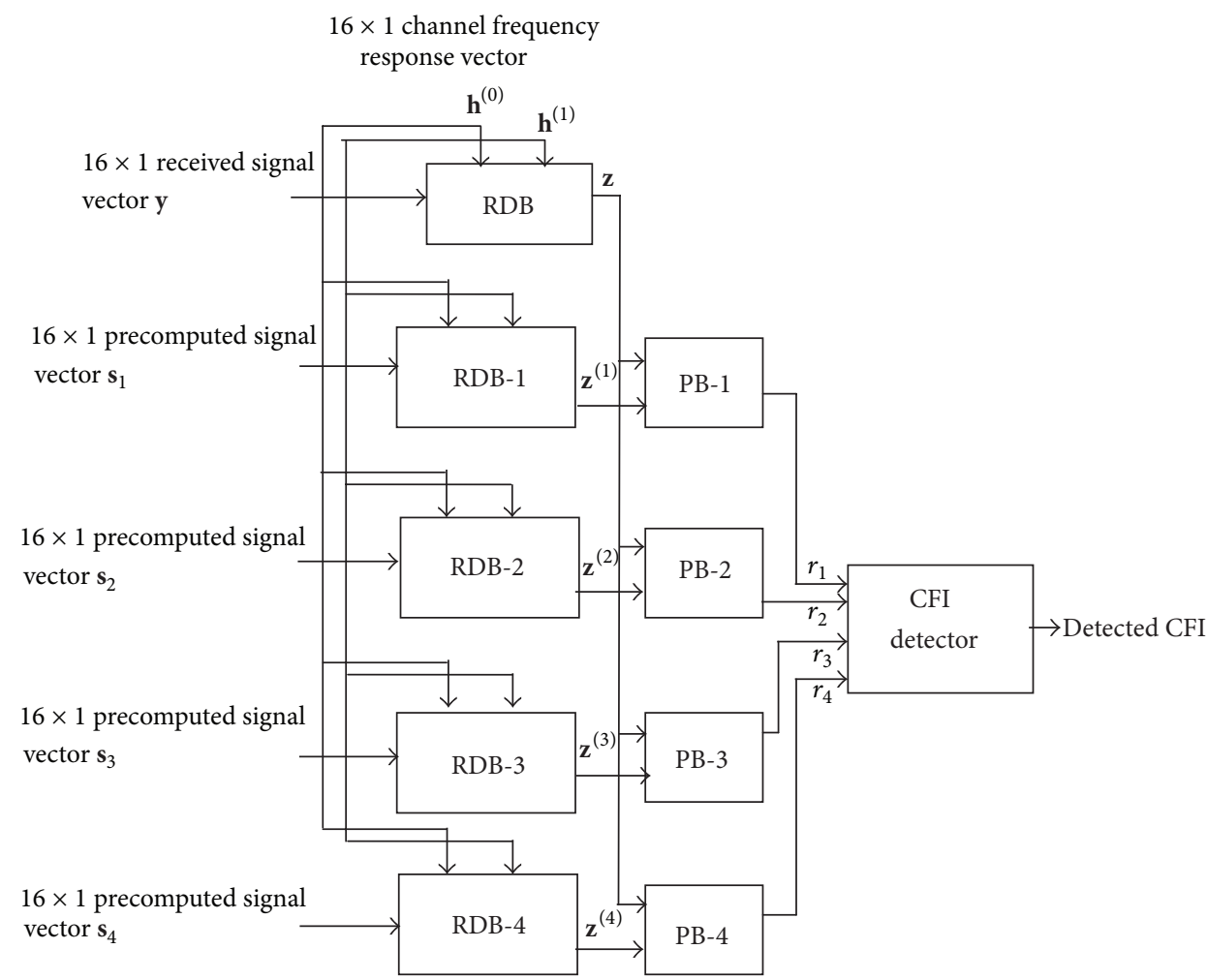

FIgURE 7: Proposed MISO receiver architecture for PCFICH.

$16 \times 1$ channel frequency response vectors $\mathbf{h}^{(0)}$ and $\mathbf{h}^{(1)}$

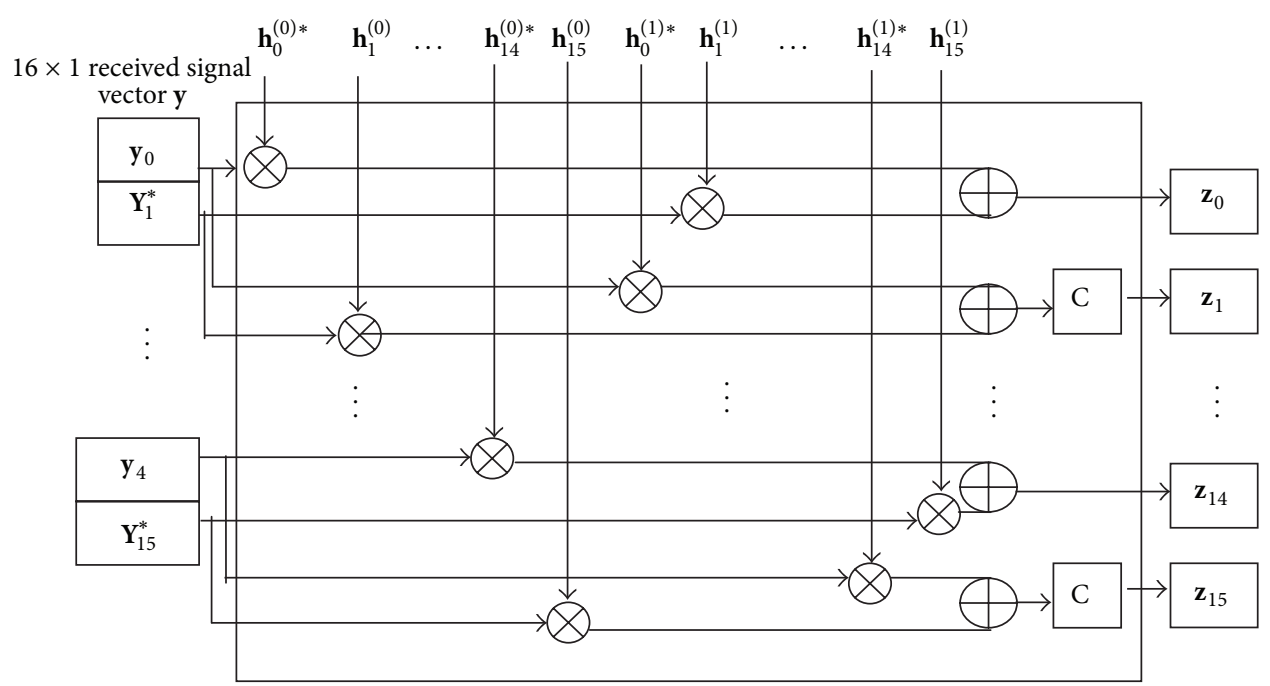

FIGURE 8: Internal architecture of MISO receiver decoding block (RDB).

the SFBC in the LTE-A standard. The precoder output at antenna port $0\left(A_{0}\right)$ and antenna port $1\left(A_{1}\right)$ is shown in Figure 6.

The notation "** represents the complex conjugate of the symbol. Basically, in precoding, a symbol $d_{0}$ from layer 0 and a symbol $d_{1}$ from layer 1 are encoded such that the antenna output is formulated using the orthogonal matrix given by

$$
A=\left[\begin{array}{cc}
d_{0} & d_{1} \\
-d_{1}^{*} & d_{0}^{*}
\end{array}\right] .
$$




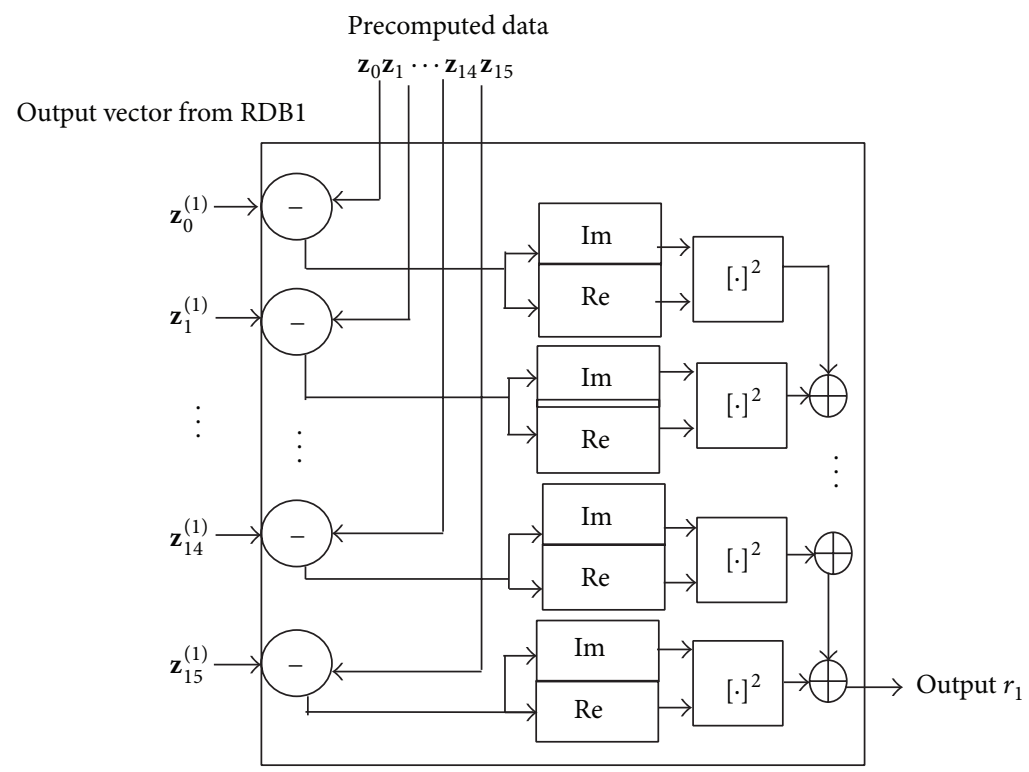

FIGURE 9: Internal architecture of MISO processing block (PB-1).

$16 \times 1$ channel frequency response vectors

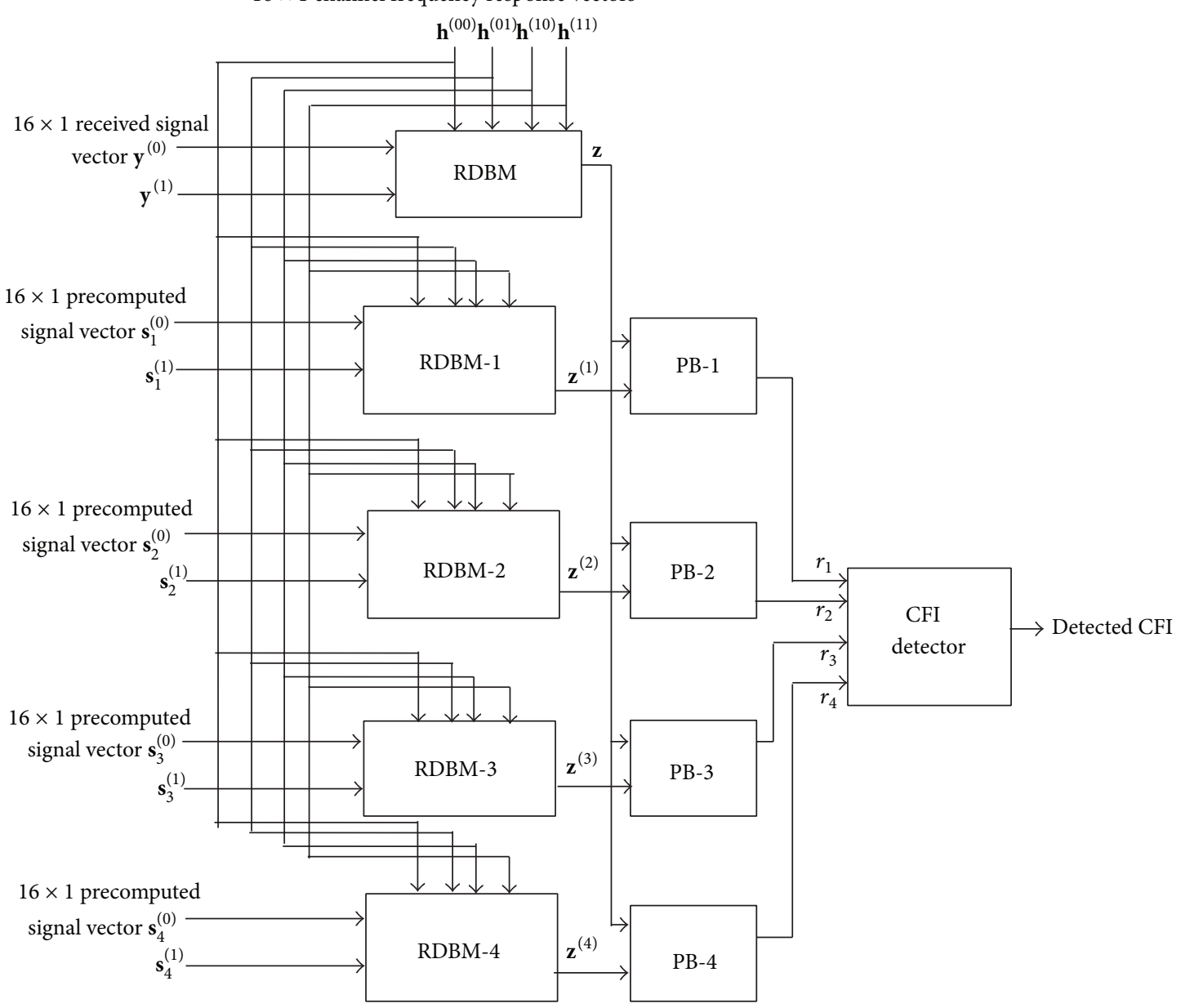

Figure 10: Proposed MIMO receiver architecture for PCFICH. 


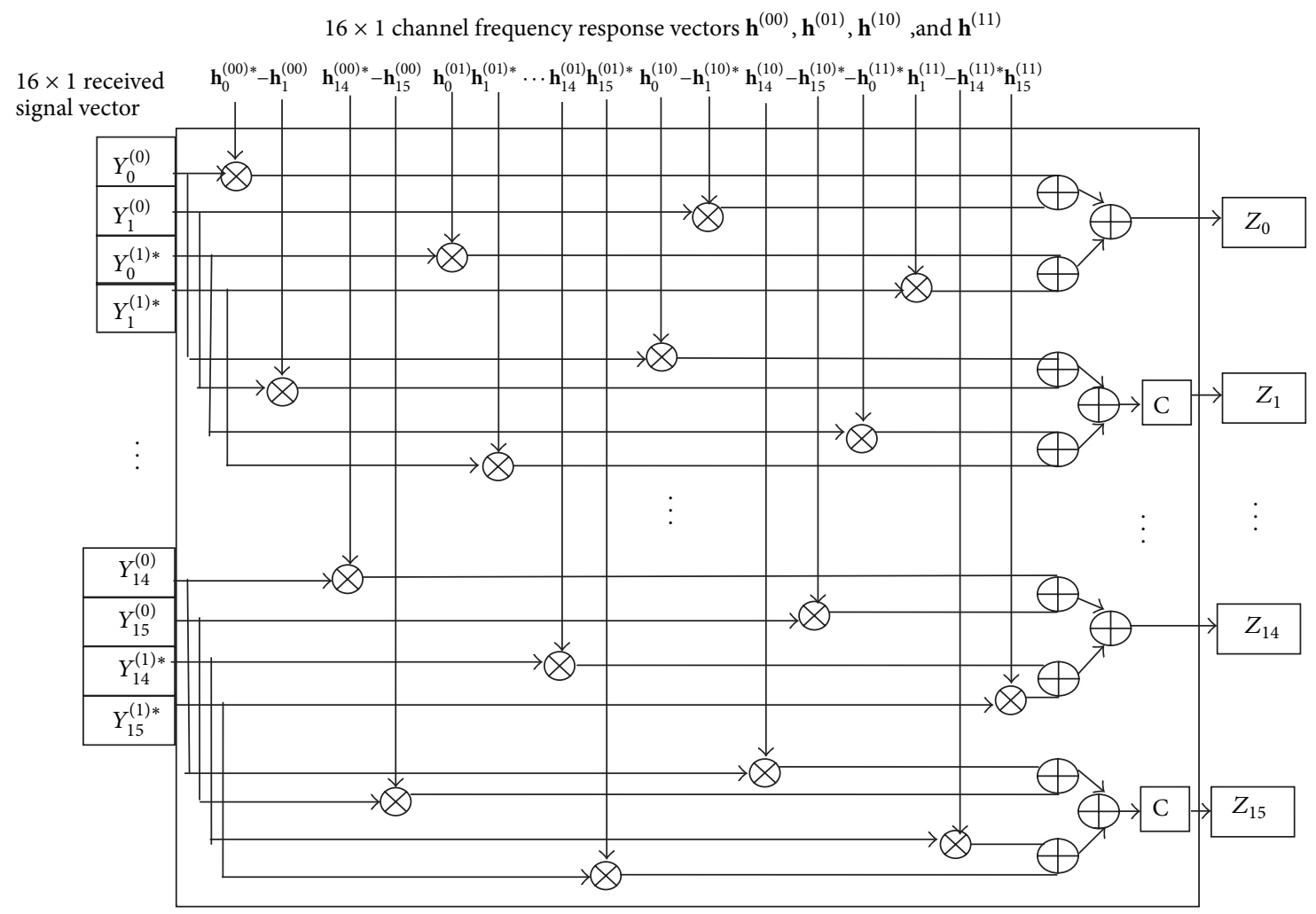

FIGURE 11: Internal architecture receiver decoding block-MIMO (RDBM).

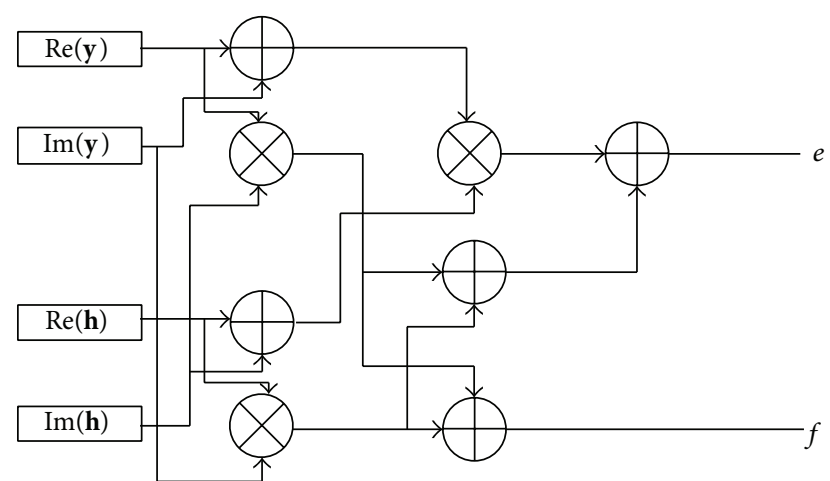

FIGURE 12: Multiplicands rearrangement for a single complex multiplication block.

This is repeated for all the 8 symbols in layer 0 and layer 1. Equation (7) defines the transmission format with the row index indicating the antenna port number and the column index indicating the subcarrier index. In $2 \times 1 \mathrm{MISO}$ configuration, the receive signals at $i$ th and $(i+1)$ th subcarrier are given in matrix form as

$$
\begin{array}{r}
{\left[\begin{array}{c}
y_{i} \\
y_{i+1}^{*}
\end{array}\right]=\left[\begin{array}{cc}
h_{i}^{(0)} & -h_{i}^{(1)} \\
h_{i+1}^{*(1)} & h_{i+1}^{*(0)}
\end{array}\right]\left[\begin{array}{c}
d_{i} \\
d_{i+1}^{*}
\end{array}\right]+\left[\begin{array}{c}
n_{i} \\
n_{i+1}^{*}
\end{array}\right],} \\
\text { for } i=0,2,4,6,8,10,12,14,
\end{array}
$$

where $h_{i}^{(0)}$ represents the channel frequency response of $i$ th subcarrier between 0th transmit antenna port and receive antenna, $d_{i}$ is data symbol at ith subcarrier, and $n_{i}$ is the noise at $i$ th subcarrier at the receive antenna. Equation (8) can simply be represented as

$$
\mathbf{y}_{i}=\mathbf{H}_{\mathrm{eff}, i} \mathbf{d}_{i}+\mathbf{n}_{i}, \quad \text { for } i=0,2,4,6,8,10,12,14,
$$

where $\mathbf{y}_{i}$ is $2 \times 1$ receive signal vector, $\mathbf{H}_{\mathrm{eff}, i}$ is the $2 \times 2$ channel matrix, $\mathbf{d}_{i}$ is $2 \times 1$ complex signal vector, and $\mathbf{n}_{i}$ is $2 \times 1$ noise vector. The objective is to detect the elements $d_{i}$ and $d_{i+1}$ of the data vector $\mathbf{d}_{i}$. Assuming that the elements of channel frequency response matrix $\mathbf{H}_{\text {eff }, i}$ are perfectly known at the receiver, the decoder output vector $\mathbf{z}_{i}$ is given by

$$
\mathbf{z}_{i}=\mathbf{H}_{\mathrm{eff}, i}^{H} \mathbf{y}_{i}, \quad \text { for } i=0,2,4,6,8,10,12,14,
$$

where $\mathbf{H}_{\mathrm{eff}, i}^{\mathrm{H}}$ is the Hermitian of the $2 \times 2$ channel transmission matrix. Equation (10) is expanded as

$$
\begin{array}{r}
{\left[\begin{array}{c}
z_{i} \\
z_{i+1}^{*}
\end{array}\right]=\left[\begin{array}{cc}
h_{i}^{*(0)} & h_{i+1}^{(1)} \\
-h_{i}^{*(1)} & h_{i+1}^{(0)}
\end{array}\right]\left[\begin{array}{c}
y_{i} \\
y_{i+1}^{*}
\end{array}\right],} \\
\quad \text { for } i=0,2,4,6,8,10,12,14 .
\end{array}
$$

The elements of decoder output are calculated as

$$
\begin{gathered}
z_{i}=h_{i}^{*(0)} y_{i}+h_{i+1}^{(1)} y_{i+1}^{*}, \quad \text { for } i=0,2,4,6,8,10,12,14, \\
z_{\mathrm{i}+1}^{*}=-h_{i}^{*(1)} y_{i}+h_{i+1}^{(0)} y_{i+1}^{*}, \quad \text { for } i=0,2,4,6,8,10,12,14 .
\end{gathered}
$$

The PCFICH receive architecture for $2 \times 1$ MISO configuration is shown in Figure 7. Receiver decoding block (RDB) 


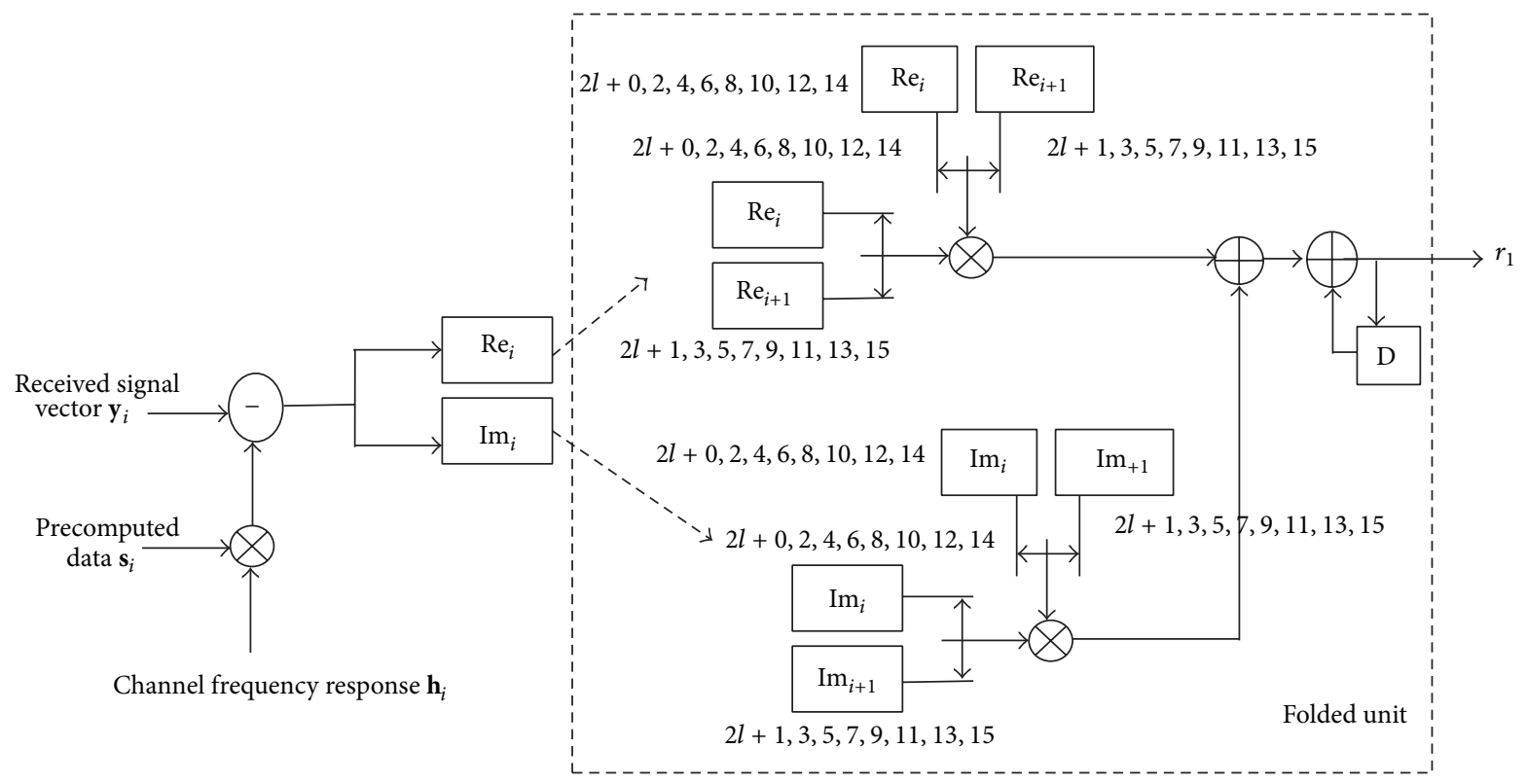

FIGURE 13: Illustration of folded architecture of RPB in SISO and SIMO.

gets the $16 \times 1$ received signal vector $y$ and computes the decoder output vector using (10), assuming that the channel frequency response vectors $\mathbf{h}^{(0)}$ and $\mathbf{h}^{(1)}$ are known. The detailed internal architecture of RDBM is shown in Figure 11. The decoder output vectors $\mathbf{z}_{i}, i=0,2,4, \ldots, 14$ are stacked as $16 \times 1$ vector $\mathbf{z}=\left[z_{0}^{T}, z_{2}^{T}, \ldots, z_{14}^{T}\right]^{T}$. The $16 \times 1$ precomputed data vectors for $\mathrm{CFI}=1,2,3,4$ are represented as $\mathbf{s}_{1}, \mathbf{s}_{2}, \mathbf{s}_{3}$, and $\mathbf{s}_{4}$ respectively.

The detailed structure of receiver decoding blocks (RDB) is shown in Figure 8. The output vectors $\mathbf{z}^{(1)}, \mathbf{z}^{(2)}, \mathbf{z}^{(3)}, \mathbf{z}^{(4)}$ from RDB-1 to RDB-4 are fed to the processing blocks (PB1 to PB-4). The detailed architecture of PB-1 is shown in Figure 9. The sum of the square magnitude of the elements of difference vector between decoded output vector $\mathbf{z}$ and the precomputed data vector $\mathbf{s}_{1}$ is the output $r_{1}$ of PB-1. Similarly $r_{2}, r_{3}$, and $r_{4}$ are computed for $\mathrm{CFI}=2,3$, and 4 using $\mathrm{PB}-$ 2 , PB-3, and PB-4, respectively. The processing block outputs $r_{1}, r_{2}, r_{3}$, and $r_{4}$ are applied to the CFI determination circuit shown in Figure 4 to detect the CFI value.

\section{System Model and Implementation Architecture for MIMO Configuration}

In MIMO system, the signals at $i$ th and $(i+1)$ th subcarrier in the receive array are given by

$$
\left[\begin{array}{c}
y_{i}^{(0)} \\
y_{i+1}^{(0)} \\
y_{i}^{(1) *} \\
y_{i+1}^{(1) *}
\end{array}\right]=\left[\begin{array}{cc}
h_{00} & h_{01} \\
h_{10} & h_{11} \\
h_{01}^{*} & -h_{00}^{*} \\
h_{11}^{*} & -h_{10}^{*}
\end{array}\right]\left[\begin{array}{c}
d_{i} \\
d_{i+1}^{*}
\end{array}\right]+\left[\begin{array}{c}
n_{i}^{(0)} \\
n_{i+1}^{(0) *} \\
n_{i}^{(1)} \\
n_{i+1}^{(1) *}
\end{array}\right],
$$

for $i=0,2,4,6,8,10,12,14$, where $h_{a b}$ represents the channel frequency response vector between $b$ th transmit antenna and $a$ th receive antenna and $n_{i}^{(j)}$ represents the noise in $i$ th subcarrier in $j$ th receive antenna. In vector form, it is written as

$$
\mathbf{y}_{i}=\mathbf{H}_{\mathrm{eff}, i} \mathbf{d}_{i}+\mathbf{n}_{i}, \quad \text { for } i=0,2,4,6,8,10,12,14,
$$

where $\mathbf{y}_{i}$ is $4 \times 1$ receive signal vector, $\mathbf{H}_{\mathrm{eff}, i}$ is the $4 \times 2$ channel frequency response vector at $i$ th and $(i+1)$ th subcarrier, $\mathbf{d}_{i}$ is $2 \times 1$ data vector at $i$ th and $(i+1)$ th subcarrier, and $\mathbf{n}_{i}$ is $4 \times 1$ noise vector. The objective is to detect the elements $d_{i}$ and $d_{i+1}$ of the data vector $\mathbf{d}_{i}$. Assuming that the elements of channel frequency response matrix $\mathbf{H}_{\text {eff }, i}$ are perfectly known at the receiver, the decoder output vector $\mathbf{z}$ is given by

$$
\mathbf{z}_{i}=\mathbf{H}_{\mathrm{eff}, i}^{H} \mathbf{y}_{i}, \quad \text { for } i=0,2,4,6,8,10,12,14,
$$

where $\mathbf{H}_{\mathrm{eff}, i}^{H}$ is the Hermitian of the $4 \times 2$ channel transmission matrix. This can be expanded as

$$
\left[\begin{array}{c}
z_{i} \\
z_{+1}^{*}
\end{array}\right]=\left[\begin{array}{cccc}
h_{00}^{*} & -h_{10}^{*} & h_{01} & h_{11} \\
h_{01}^{*} & -h_{11}^{*} & -h_{00} & -h_{10}
\end{array}\right]\left[\begin{array}{c}
y_{i}^{(0)} \\
y_{i+1}^{(0)} \\
y_{i}^{(1) *} \\
y_{i+1}^{(1) *}
\end{array}\right],
$$

for $i=0,2,4,6,8,10,12,14$.

The decoder outputs are given by

$$
\begin{array}{r}
z_{i}=h_{00}^{*} y_{i}^{(0)}-h_{10}^{*} y_{i+1}^{(0)}+h_{01} y_{i}^{(1) *}+h_{11} y_{i+1}^{(1) *}, \\
\text { for } i=0,2,4,6,8,10,12,14 . \\
z_{i+1}^{*}=h_{01}^{*} y_{i}^{(0)}-h_{11}^{*} y_{i+1}^{(0)}-h_{00} y_{i}^{(1) *}-h_{10} y_{i+1}^{(1) *},
\end{array}
$$

for $i=0,2,4,6,8,10,12,14$. 


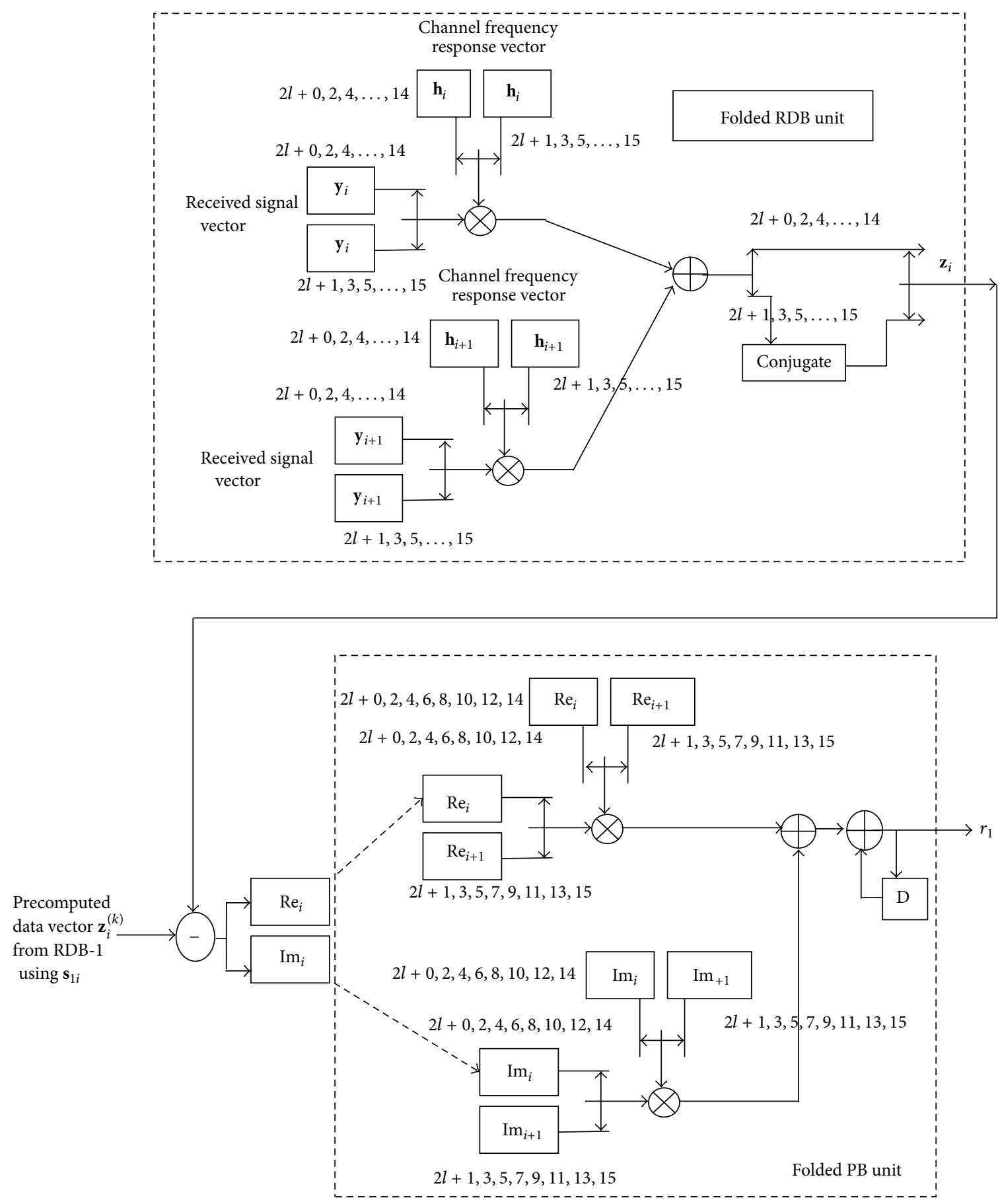

FIGURE 14: Illustration of proposed architecture for RDB and PB in MISO and MIMO. Note: receiver decoding block (RDB) in MISO is termed as RDBM in MIMO.

The PCFICH receiver architecture of $2 \times 2$ MIMO configurations is shown in Figure 10.

Receiver decoding block $(\mathrm{RDBM})$ gets the $16 \times 1$ received signal vector $y$ and computes the decoder output vector using (14), assuming that the channel frequency response vectors $\mathbf{h}^{(00)}, \mathbf{h}^{(01)}, \mathbf{h}^{(10)}$, and $\mathbf{h}^{(11)}$ are known. The $16 \times 1$ precomputed data vectors for $\mathrm{CFI}=1,2,3$, and 4 are represented as $\mathbf{s}_{1}^{(0)}$, $\mathbf{s}_{2}^{(0)}, \mathbf{s}_{3}^{(0)}$, and $\mathbf{s}_{4}^{(0)}$, respectively, for antenna 0 , and as $\mathbf{s}_{1}^{(1)}$, $\mathbf{s}_{2}^{(1)}, \mathbf{s}_{3}^{(1)}$, and $\mathbf{s}_{4}^{(1)}$, respectively, for antenna 1 . The received signal vectors $\mathbf{y}_{i}^{(0)}$ and $\mathbf{y}_{i}^{(1)}$ multiply with the four channel estimation vectors to give decoded output vector $\mathbf{z}$ that is sent to the processing block $(\mathrm{PB})$ which is shown in Figure 9. The decoder outputs $z_{i}, i=0,2,4, \ldots, 14$ are stacked as $16 \times 1$ vector $\mathbf{z}=\left[z_{0}^{T}, z_{2}^{T}, \ldots, z_{14}^{T}\right]^{T}$. Similarly, RDBM1 gives output vector $\mathbf{z}^{(1)}$ using the precomputed data vectors $\mathbf{y}_{1}^{(0)}$ and $\mathbf{y}_{1}^{(1)}$ and channel estimation vectors. The architecture of PBs and 


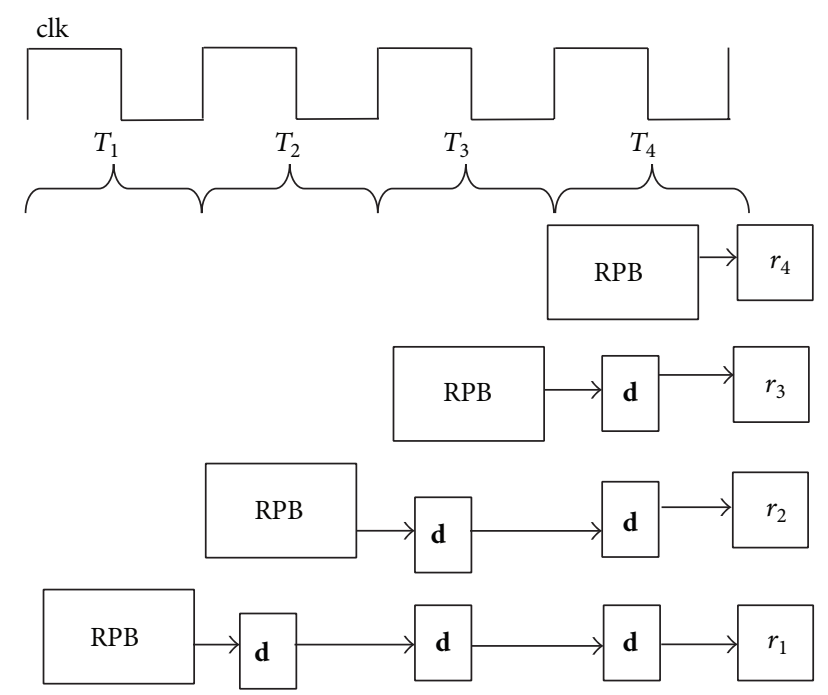

FIGURE 15: Illustration of superscalar method for SISO and SIMO (with no complex multiplications and operating from $T_{1}$ to $T_{4}$ ).

the CFI detection architecture are similar to that of the MISO system. The sum of the squared magnitude of the difference between each element in the decoded output vector $\mathbf{z}$ and its precomputed data in the vector $\mathbf{z}^{(1)}$ is the output $r_{1}$ of PB1. Similarly $r_{2}, r_{3}$, and $r_{4}$ are computed for other CFI. The $r_{1}, r_{2}$, $r_{3}$, and $r_{4}$ are compared to determine the minimum value by the CFI detector shown in Figure 4.

\section{PCFICH Receiver Implementation Methods}

The PCFICH receiver architectures can be implemented directly based on the basic architectures developed in Sections 3 and 4. But, in order to effectively utilize the resources in FPGA, the implementation of basic architectures is done using the modified novel architectures based on VLSI DSP techniques, namely, folding and superscalar processing approach.

5.1. Direct Implementation with Multiplicands Rearranged Method. In the receiver architecture for SISO and SIMO, the $16 \times 1$ received signal vector is directly subtracted from the precomputed data vector for a given CFI. This requires lesser number of multipliers and adders when compared to MISO and MIMO. In MISO and MIMO configurations, complex multiplications are necessary for the multiplication of $\mathbf{H}_{\text {eff }}^{H}$ with the received signal vector. It increases the number of multiplications in the CFI detection process. Hence, optimum rearrangement of the terms is carried out to minimize the number of multiplications. Further, the intermediate products are reused in the calculation of real and imaginary parts. Consider the multiplication of two complex numbers $\operatorname{Re}\{h\}+j \operatorname{Im}\{h\}$ and $\operatorname{Re}\{y\}+j \operatorname{Im}\{y\}$. The output real part $(e)$ and imaginary part $(f)$ terms are given by

$$
\begin{aligned}
& e=\operatorname{Re}\{h\} \operatorname{Re}\{y\}-\operatorname{Im}\{h\} \operatorname{Im}\{y\}, \\
& f=\operatorname{Re}\{h\} \operatorname{Im}\{y\}+\operatorname{Im}\{h\} \operatorname{Re}\{y\} .
\end{aligned}
$$

It requires four multiplications and two additions. To reduce the number of multiplications, the terms in (18) are rearranged as

$$
\begin{gathered}
e=[\operatorname{Re}\{y\}-\operatorname{Im}\{y\}][\operatorname{Re}\{h\}-\operatorname{Im}\{h\}] \\
-\operatorname{Re}\{y\} \operatorname{Im}\{h\}+\operatorname{Im}\{y\} \operatorname{Re}\{h\}, \\
f=\operatorname{Re}\{y\} \operatorname{Im}\{h\}+\operatorname{Im}\{y\} \operatorname{Re}\{h\} .
\end{gathered}
$$

Since the terms $\operatorname{Re}\{y\} \operatorname{Im}\{h\}$ and $\operatorname{Im}\{y\} \operatorname{Re}\{h\}$ are in (19), it requires only three multiplications but five additions. This kind of rearrangement of the multiplicands is employed in the processing blocks at the cost of increased additions as shown in Figure 12.

5.2. Proposed Architecture Using Folding Method. Folding architecture systematically determines the control circuits in DSP architectures where multiple algorithmic operations are time-multiplexed to a single functional unit [12]. It is used for synthesis of DSP architectures that can be operated at single or multiple clocks. It reduces the number of hardware functional units (FUs) by a factor of $N$ at the expense of increased computation time.

The folding architecture is introduced in the receiver structure of RPB in SISO and SIMO configurations and of $\mathrm{RPB}$ and PB in MISO and MIMO configurations as shown in Figures 13 and 14, respectively. For SISO RPB, there are 16 hardware lines to calculate the value of $r_{1}$ each requiring two multipliers. Hence the number of multipliers used in one $\mathrm{RPB}$ is 32 . In order to reduce the number of multipliers and adders, folding architecture is proposed. This architecture uses only two multipliers and performs the operation of a single hardware line 16 times in sequential way. The difference between the product of channel frequency response vector with the precomputed data vector and the received signal vector is stored in registers. At a time, one resultant signal pair involves in computation using two multipliers to get the value of $z_{i}$. Four switches operating in system clock speed are involved in the architecture where two switches are used to pass the real part of the signal to one multiplier, while the other two switches are used to pass the imaginary part of the signal to another multiplier. The multipliers pass the products to the first adder for $z_{i}$. The output of the first adder is passed to the second adder with a delay to accumulate the values $z_{0}$ to $z_{15}$ into a register in subsequent clock cycles. This process requires 16 clock cycles and the CFI is detected at the 17th clock cycle. Though it takes longer time for the clock cycles to get the output, the resources are minimized in this method.

The folded architecture of decoding block of MISO and MIMO involving complex multiplication of the channel frequency response vector and the receive signal vector is shown in Figure 14. There are 2 complex multiplications and one addition in each of the 16 hardware lines. Hence total resource elements used are 32 complex multiplications and 16 additions. The folded architecture which reduces to just 2 complex multiplications and one addition requires five switches. Two switches are used to pass the first element of the receive signal vector and its corresponding channel 

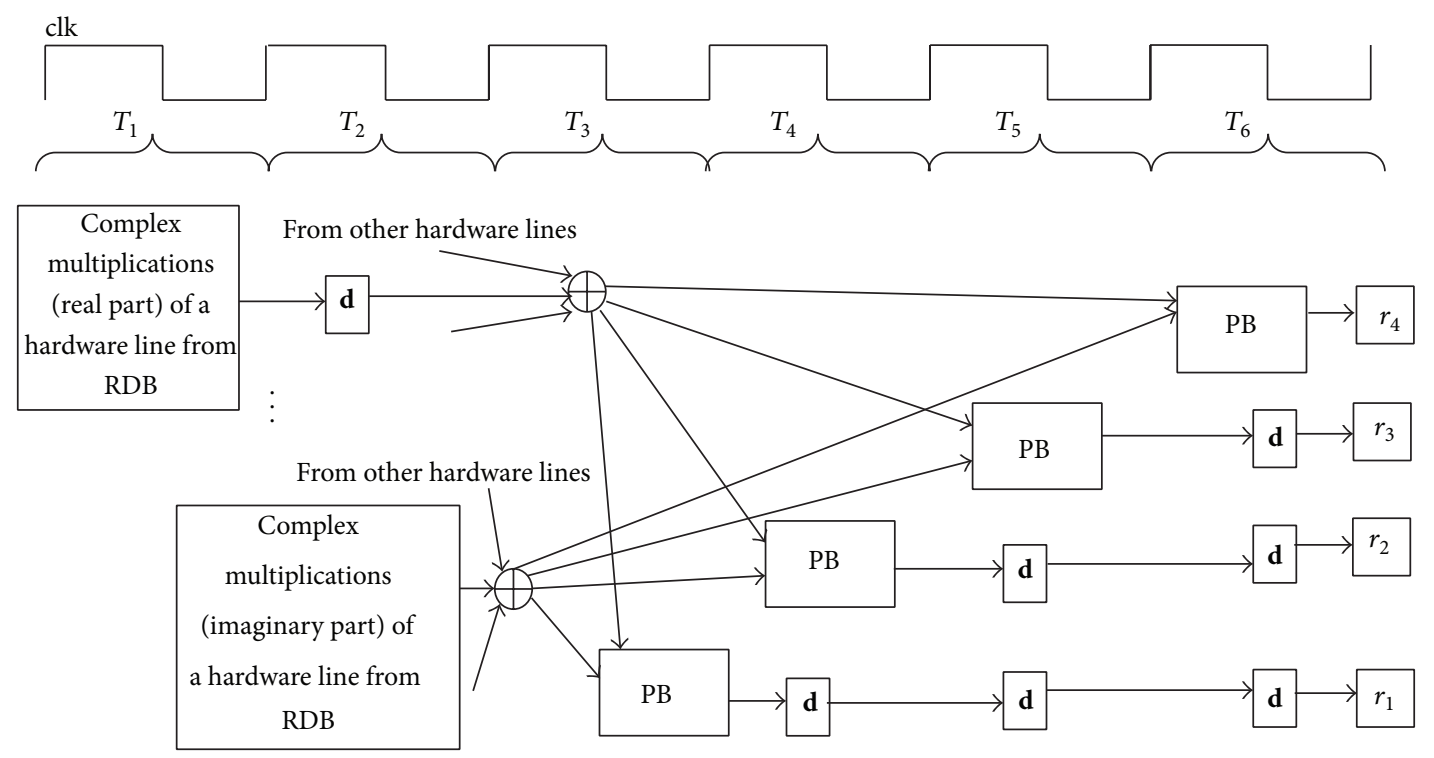

FIGURE 16: Illustration of superscalar method for MISO and MIMO (with complex multiplications and operating from $T_{1}$ to $T_{6}$ ).

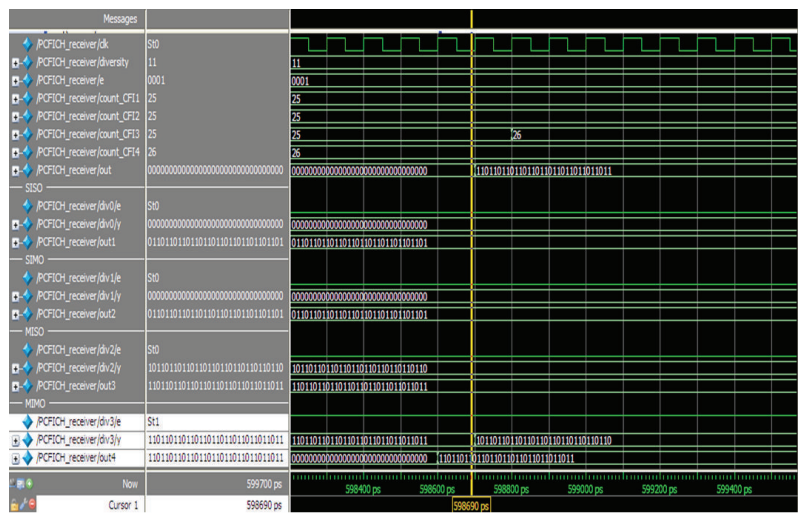

FIGURE 17: Simulation waveform for PCFICH receiver.

frequency response vector to one multiplier and other two switches are used to pass the second element of receive signal vector and its channel frequency response vector to another multiplier. These four switches operate in system clock speed. The multipliers pass their products to the adder through the fifth switch before moving to $\mathrm{PB}$. This process requires 16 clock cycles and the CFI is detected at the 17th clock cycle.

\subsection{Proposed Architecture Using Superscalar Method. Super-} scalar approach is another low resource utilizing VLSI DSP technique. The superscalar processing method includes parallel processing and pipelining strategies. In this case, parallel operation for the 16 pairs of hardware lines is arranged with pipelining of the subtraction and square magnitude operations for each CFI. SISO configuration does not have complex multiplications and it has only square magnitude operations. Hence the RPB of SISO has 16 hardware lines each having 2 multipliers which results to a total of 32 multipliers.
This setup requires more hardware resources than folding, but the output is obtained at every 4 th clock cycle as shown in Figure 15. SIMO configuration which involves two receive antenna signal processing, requires twice the number of multiplications as that of SISO and the output is obtained at every 4 th clock cycle. The block " $d$ " represents the delay element introduced to buffer the values and produce the outputs at the same time instant.

For MISO configuration the RDB has 16 hardware lines, with 2 complex multiplications each. Since each complex multiplication requires four real multiplications, RDB can be executed in two clock cycles by reusing 64 multipliers. 32 multipliers are required for PB taking 4 clock cycles. Hence 96 multipliers are required in MISO configuration. For MIMO configuration, the $\mathrm{RDB}$ requires reuse of 128 multipliers taking 2 clock cycles and an additional 32 multipliers are required for the PB taking 4 clock cycles. Hence 160 multipliers are required for MISO configuration and the output is obtained at every 6th clock cycle as shown in the Figure 16. The block " $d$ " represents the delay element introduced to buffer the values and produce the outputs at the same time instant.

\section{Results and Discussion}

The proposed receiver architectures for PCFICH in SISO, SIMO, MISO, and MIMO configurations are implemented using the Xilinx PlanAhead tool on the Virtex-6 FPGA xc6vlx240tffl156-1 device board. The target device Virtex-6 has only 768 DSP elements. Table 2 shows the performance of the proposed architectures using folding and superscalar methods being compared with the direct implementation of PCFICH receiver, in terms of resource utilisation, speed, and power for all the SISO, SIMO, MISO, and MIMO 


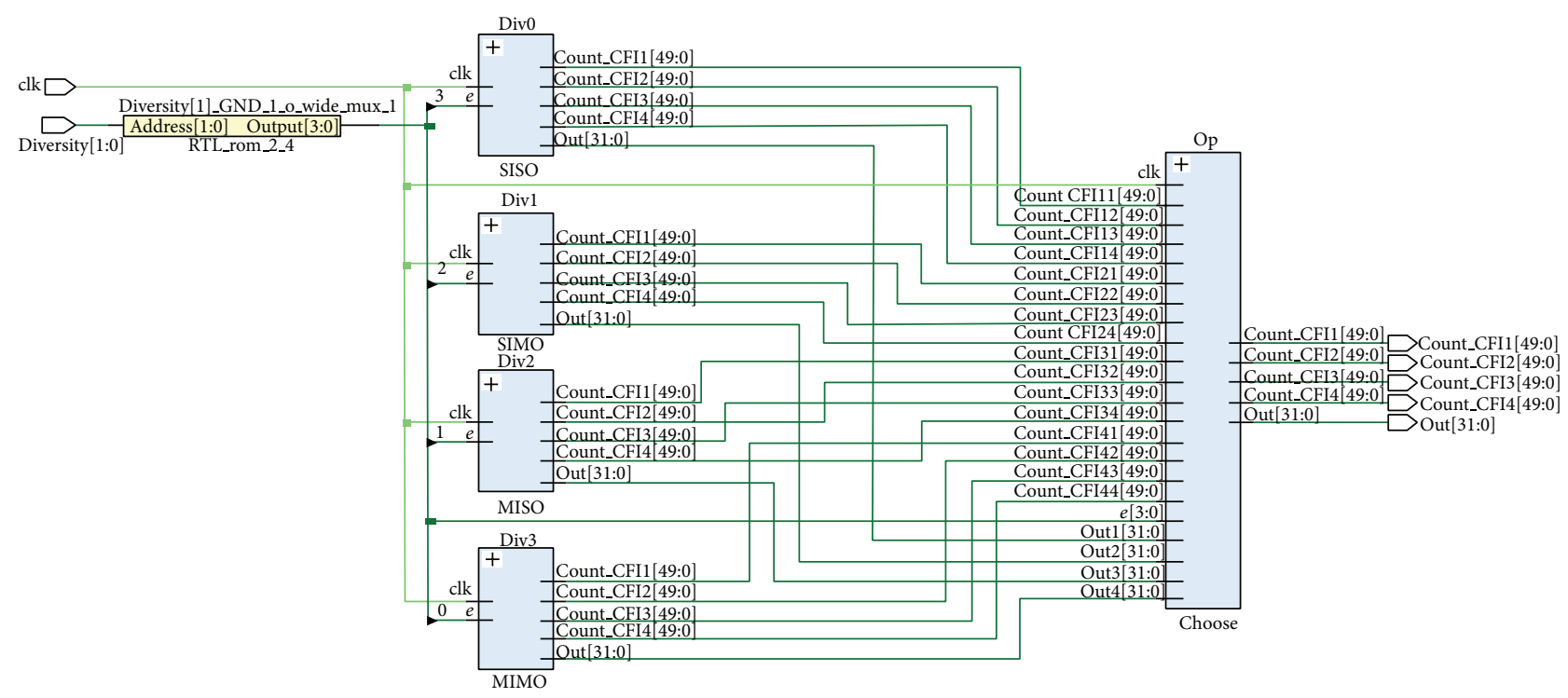

FIgURE 18: RTL schematic for combined PCFICH architecture with diversity.

TABLE 2: Performance of proposed architectures based on folding and superscalar method.

\begin{tabular}{|c|c|c|c|c|c|c|c|c|}
\hline Diversity & Method & Multipliers & Adders & DSP elements & LUTs & Total delay (ns) & Speed $(\mathrm{MHz})$ & Dynamic power \\
\hline \multirow{3}{*}{ SISO } & Direct & 125 & 245 & 125 & 5479 & 39.081 & 25.587 & - \\
\hline & Folding (16T) & 8 & 81 & 16 & 2561 & 114.448 & 8.737 & 84 \\
\hline & Superscalar (4T) & 32 & 182 & 66 & 2731 & 69.333 & 14.423 & 93 \\
\hline \multirow{3}{*}{ SIMO } & Direct & 250 & 494 & 250 & 10942 & 40.278 & 24.827 & - \\
\hline & Folding (16T) & 16 & 165 & 32 & 5117 & 130.704 & 7.651 & 159 \\
\hline & Superscalar $(4 \mathrm{~T})$ & 64 & 318 & 130 & 5623 & 53.873 & 18.562 & 170 \\
\hline \multirow{3}{*}{ MISO } & Direct & 224 & 580 & 594 & 14880 & 43.023 & 23.243 & - \\
\hline & Folding (16T) & 14 & 101 & 39 & 3950 & 255.264 & 3.917 & 173 \\
\hline & Superscalar (6T) & 96 & 338 & 196 & 6156 & 80.495 & 12.423 & 208 \\
\hline \multirow{3}{*}{ MIMO } & Direct & 320 & 844 & 675 & 17380 & 56.962 & 17.555 & - \\
\hline & Folding (16T) & 20 & 155 & 46 & 4395 & 256.528 & 3.898 & 374 \\
\hline & Superscalar (6T) & 160 & 465 & 262 & 6932 & 85.822 & 11.652 & 382 \\
\hline
\end{tabular}

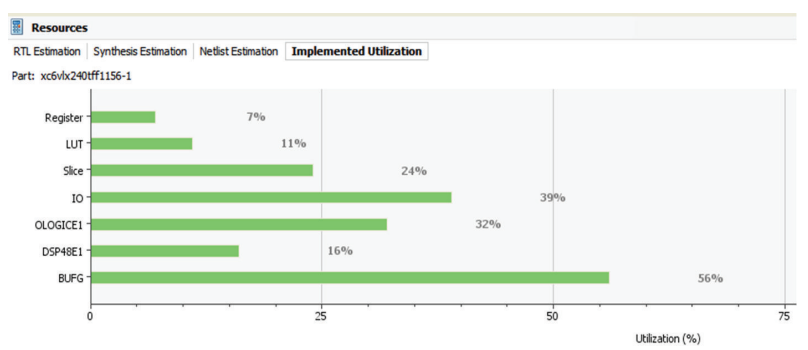

FIGURE 19: Resource utilization graph for generalized architecture.

configurations. The proposed architectures based on folding and superscalar processing methods require less number of resource elements.

In the folding approach, resource utilization is less compared to the direct and superscalar approach at the cost of reduced speed of operation but it is suitable for real-time frame timings. When the LTE-A system operates at $1.4 \mathrm{MHz}$ bandwidth, maximum time available for detection at each subcarrier is $992.063 \mathrm{~ns}$ since each slot of $0.5 \mathrm{~ms}$ duration in a frame (10 ms radio frame duration) consists of 7 OFDM symbols and there are 72 subcarriers along one OFDM symbol. The total delay in the receiver architecture is within the LTE time constraint. The dynamic power consumption is less in the folding method compared to superscalar method due to decrease in block arithmetic. Direct method does not require sequential execution and clocking and hence total power consumption is due to static power. Hence, it is inferred that the proposed architecture based on folding method is more suitable for CFI detection. The simulation waveform of the proposed architecture based on folding method is shown in Figure 17 for SISO, SIMO, MISO, and MIMO configurations.

A general architecture based on folding method which operates at all the four SISO, SIMO, MISO, and MIMO configurations has also been developed. In this architecture, 
TABLE 3: Resource requirements of proposed architecture using folding method.

\begin{tabular}{lcccccc}
\hline Parameter & Multipliers & Adders & Minimum clock period (ns) & Total delay $(\mathrm{ns})$ & Speed $(\mathrm{MHz})$ & Total dynamic power $(\mathrm{mW})$ \\
\hline Value & 58 & 518 & 16.033 & 256.528 & 3.898 & 1019 \\
\hline
\end{tabular}

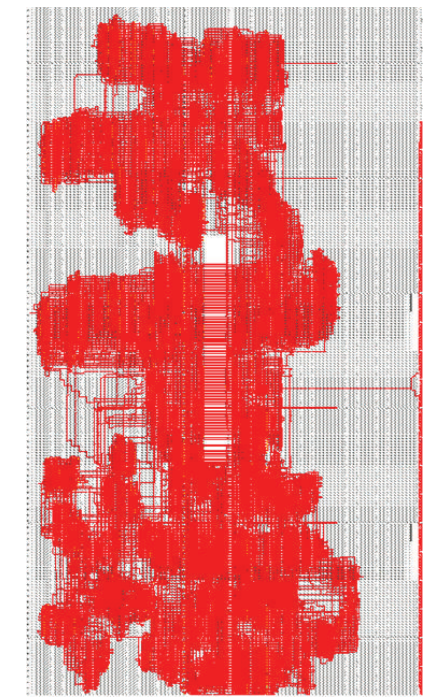

FIGURE 20: Implemented device in FPGA editor.

a control variable " $e$ " is used to enable or disable the submodules SISO, SIMO, MISO, or MIMO according to the selection input "diversity." CFI is detected at every 17 th clock cycle. The synthesis results of a general architecture based on folding show that it utilizes minimum resources in XC6VLX240TFF1156-1 Virtex 6 device (768 DSPs). This is summarized in Table 3. Dynamic power consumption is due to internal switching contributed by the clock $(246 \mathrm{~mW})$, logic $(670 \mathrm{~mW})$, and the block arithmetic $(103 \mathrm{~mW})$.

Figure 18 shows the RTL schematic of 4 diversity blocks "div0," "div1," "div2," and "div3" corresponding to SISO, SIMO, MISO, and MIMO controlled by wires named "e." Power consumed includes both static power and dynamic power due to internal switching.

Figure 19 shows the resource utilization graph which shows the percentage of registers, lookup tables (LUTs), slices, DSP elements, and buffers used.

Figure 20 shows the implemented device in FPGA editor with the implemented components and interconnections between the components configured into the FPGA device.

\section{Conclusion}

In this paper, low complexity, low resource single, or multiantenna CFI detection at the receiver system has been proposed and analyzed using modelsim and implementation in the Virtex-6 device in Xilinx PlanAhead tool. In the receiver, computational complexity and the resource utilization are minimized by employing arithmetic operational rearrangement and suboptimal sequential DSP algorithm called the folding approach. The proposed architecture using folding method complies with the LTE frame timing constraint in SISO, SIMO, MISO and MIMO configurations. It is a suitable solution for the area optimized hardware implementation of receiver structures for PCFICH. In future, a total hardware accommodating all the physical downlink control channels of the 3GPP-LTE-A with low resource utilization could be synthesized and implemented.

\section{Conflict of Interests}

The authors do not have direct financial relation with any commercial identity mentioned in the paper or any other conflict of interests.

\section{Acknowledgments}

The authors wish to express their sincere thanks to All India Council for Technical Education, New Delhi, for the Grant to do the Project titled Design of Testbed for the Development of Optimized Architectures of MIMO Signal Processing (no. 8023/RID/RPS/039/11/12). They are also thankful to the Managements of Mepco Schlenk Engineering College, Sivakasi, and Thiagarajar College of Engineering, Madurai, for their constant support and encouragement to carry out this research work successfully.

\section{References}

[1] 3GPP TS 36. 211 Version 11. 0. 0 Release 11 (2012-10) Evolved Universal Terrestrial Radio Access (E-UTRA), Physical Channels and Modulation, 2012.

[2] J. Ketonen and M. Juntti, "SIC and K-best LSD receiver implementation for a MIMO-OFDM system," in Proceedings of the 16th European Signal Processing Conference (EUSIPCO '08), Lausanne, Switzerland, August 2008.

[3] J. Ketonen, M. Juntti, and J. R. Cavallaro, "Performancecomplexity comparison of receivers for a LTE MIMOOFDM system," IEEE Transactions on Signal Processing, vol. 58, no. 6, pp. 3360-3372, 2010.

[4] X. Huang, C. Liang, and J. Ma, "System architecture and implementation of MIMO sphere decoders on FPGA," IEEE Transactions on Very Large Scale Integration Systems, vol. 16, no. 2, pp. 188-196, 2008.

[5] Y. Lin, Y. Chen, C. Chu, C. Zhan, and A. Wu, "Dual-mode low-complexity codebook searching algorithm and VLSI architecture for LTE/LTE-advanced systems," IEEE Transactions on Signal Processing, vol. 61, no. 14, pp. 3545-3562, 2013.

[6] D. Wu, J. Eilert, R. Asghar, and D. Liu, "VLSI implementation of a fixed-complexity soft-output MIMO detector for high-speed wireless," EURASIP Journal on Wireless Communications and Networking, vol. 2010, Article ID 893184, 13 pages, 2010.

[7] C. Mehlführer, M. Wrulich, J. C. Ikuno, D. Bosanska, and M. Rupp, "Simulating the long term evolution physical layer," in 
Proceedings of the 17th European Signal Processing Conference (EUSIPCO '09), pp. 1471-1478, Glasgow, UK, August 2009.

[8] S. J. Thiruvengadam and L. M. A. Jalloul, "Performance analysisof the 3GPP-LTE physical control channels," EURASIP Journalon Wireless Communications and Networking, vol. 2010, Article ID 914934, 10 pages, 2010.

[9] S. M. Alamouti, "A simple transmit diversity technique for wireless communications," IEEE Journal on Selected Areas in Communications, vol. 16, no. 8, pp. 1451-1458, 1998.

[10] S. S. A. Abbas and S. J. Thiruvengadam, "FPGA implementation of 3GPP-LTE-A physical downlink control channel using diversity techniques," International Journal of Wireless and Mobile Computing, vol. 9, no. 2, p. 84, 2013.

[11] S. Ahmadi, LTE-Advanced: A Practical Systems Approach to Understanding 3GPP LTE Releases 10 and 11 Radio Access Technologies, Sassan Ahmadi Academic Press, 2013.

[12] K. K. Parhi, VLSI Digital Signal Processing Systems-Design and Implementation, Wiley-Interscience, 1999. 

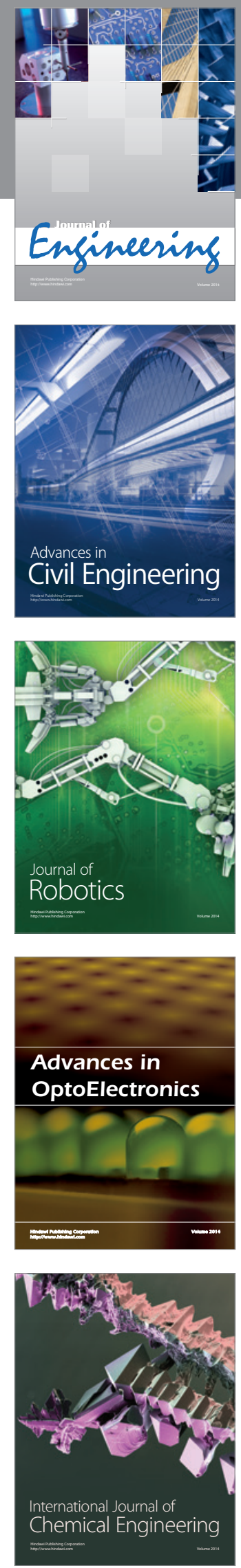

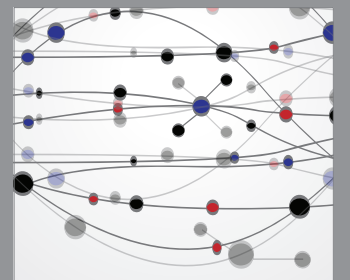

The Scientific World Journal
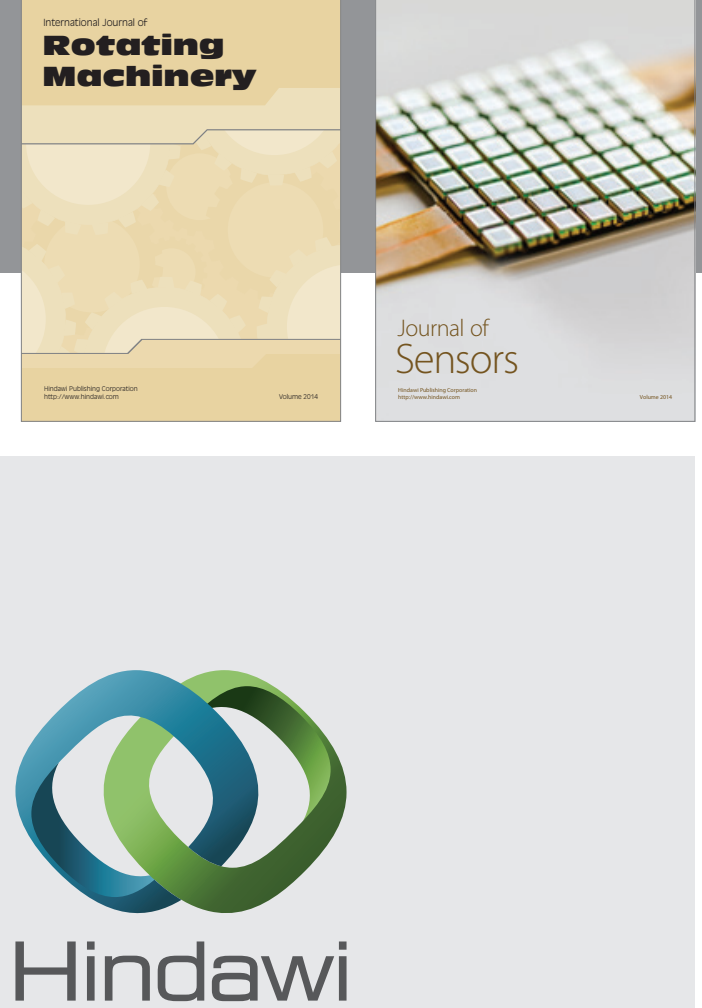

Submit your manuscripts at http://www.hindawi.com
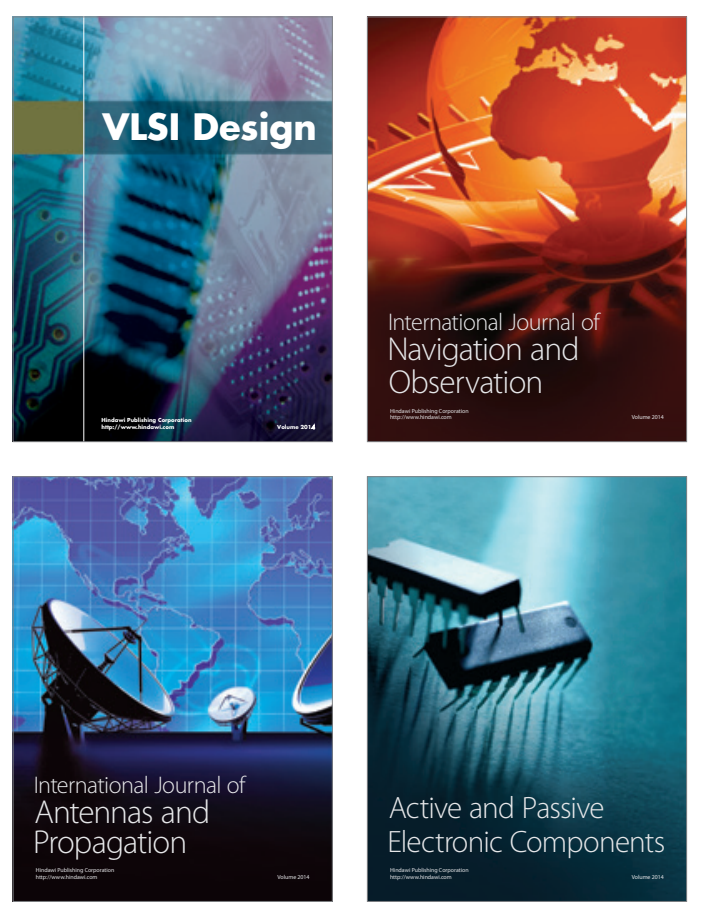
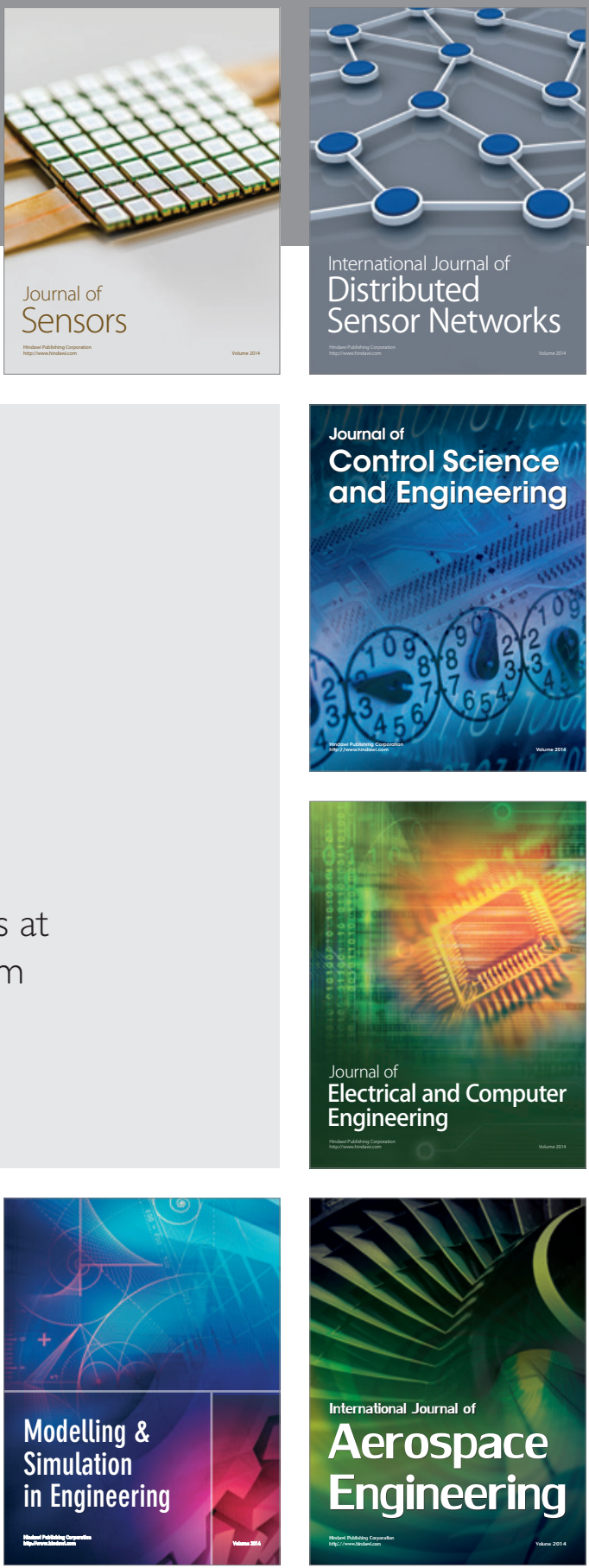

Journal of

Control Science

and Engineering
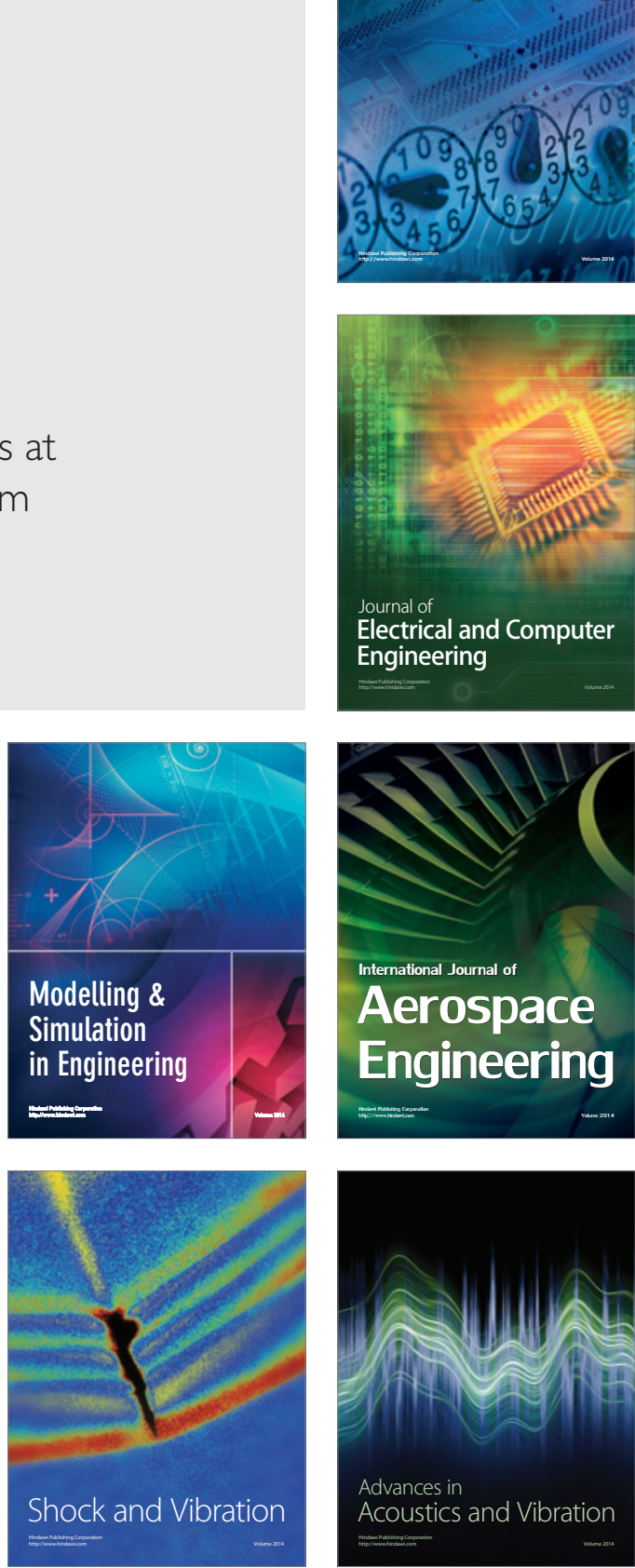